\title{
Eigenvalue distribution of some fractal semi-elliptic differential operators
}

\section{Walter Farkas}

Universität München, Mathematisches Institut, Theresienstrasse 39, 80333 München, Germany (e-mail: farkas@rz.mathematik.uni-muenchen.de)

Received September 15, 1999; in final form January 24, 2000 /

Published online December 8, 2000 - (C) Springer-Verlag 2000

Abstract. We consider differential operators of type

$$
\begin{aligned}
A u(x) & =u(x)+(-1)^{t_{1}} \frac{\partial^{2 t_{1}} u(x)}{\partial x_{1}^{2 t_{1}}}+(-1)^{t_{2}} \frac{\partial^{2 t_{2}} u(x)}{\partial x_{2}^{2 t_{2}}}, \\
x & =\left(x_{1}, x_{2}\right) \in \mathbb{R}^{2}
\end{aligned}
$$

and Sierpinski carpets $\Gamma \subset \mathbb{R}^{2}$.

The aim of the paper is to investigate spectral properties of the fractal differential operator $A^{-1} \circ \operatorname{tr}^{\Gamma}$ acting in the anisotropic Sobolev space $W_{2}^{\left(t_{1}, t_{2}\right)}\left(\mathbb{R}^{2}\right)$ where $\operatorname{tr}^{\Gamma}$ is closely related to the trace operator $t r_{\Gamma}$.

Mathematics Subject Classification (1991): 35P15, 46E35, 28A90

\section{Introduction}

Let $\Omega$ be a bounded domain in $\mathbb{R}^{n}$ having $C^{\infty}$ boundary $\partial \Omega$, let $(-\Delta)^{-1}$ be the inverse of the Dirichlet Laplacian in $\Omega$, let $W_{2}^{1}(\Omega)$ be the usual Sobolev space, and let $\stackrel{\circ}{W}_{2}^{1}(\Omega)=\left\{u \in W_{2}^{1}(\Omega): u \mid \partial \Omega=0\right\}$.

Let $\Gamma$ be some compact fractal with $|\Gamma|=0$ and $\Gamma \subset \Omega$. The operator $\operatorname{tr}^{\Gamma}$, closely related to the trace operator $\operatorname{tr}_{\Gamma}$, is defined by

$$
\left(\operatorname{tr}^{\Gamma} f\right)(\varphi)=\int_{\Gamma}\left(\operatorname{tr}_{\Gamma} f\right)(\gamma)(\varphi \mid \Gamma)(\gamma) d \mu(\gamma), \quad \varphi \in D(\Omega),
$$

where $\mu$ is the Radon measure underlying the fractal $\Gamma$. 
The distribution of the eigenvalues of the operator

$$
B=(-\Delta)^{-1} \circ \operatorname{tr}^{\Gamma}
$$

acting in the Sobolev space $\stackrel{\circ}{W}_{2}^{1}(\Omega)$ is a topic extensively treated in the book [32] of $\mathrm{H}$. Triebel.

These investigations are motivated in a natural way by the so-called fractal drums. If $\lambda_{k}, k \in \mathbb{N}$, are the positive eigenvalues of $(-\Delta)^{-1} \circ \operatorname{tr}^{\Gamma}$, ordered by magnitude and counted with respect to their multiplicities, then $\eta_{k}=\lambda_{k}^{-\frac{1}{2}}$ are the eigenfrequencies of a vibrating membrane (interpreted as the bounded domain $\Omega$ ), fixed at its boundary, having the whole mass concentrated on the fractal $\Gamma \subset \Omega$.

We want to mention here that the notion of fractal drums has several meanings. As for the intention described above (which is that from [32]) to study fractal membranes in smooth domains, we know only a few further papers in literature, see T. Fujita [14], K. Naimark and M. Solomyak [20] and [21], M. Solomyak and E. Verbitsky [26], and the more recent article of D. E. Edmunds and H. Triebel [5].

Further results on the vibrations of "fractal drums" are obtained in different settings. Maybe the best known version is connected with the study of the Laplacian on a fractal, as it is done for example in the works of J. Kigami and M. L. Lapidus, see [16] and [17] and the relevant references therein.

A detailed discussion on these different aspects concerning fractal drums can be found in [32], especially in Sects. 26.2 and 30.1-30.5, where one can find also comprehensive references extending the subject.

Let $0<d<n$. Then $\Gamma$ is called a $d$-set if there are a Radon measure $\mu$ in $\mathbb{R}^{n}$ and two positive numbers $c_{1}$ and $c_{2}$ such that for all balls $B(\gamma, r)$ centred at $\gamma \in \Gamma$ and of radius $0<r<1$,

$$
\text { supp } \mu=\Gamma \quad \text { and } \quad c_{1} r^{d} \leq \mu(B(\gamma, r)) \leq c_{2} r^{d} .
$$

The notion of (isotropic) $d$-set occurs both in the theory of function spaces and in fractal geometry, see [15], [32], [6], [7].

Considering $\Gamma$ a $d$-set with $n-2<d<n(0<d<1$ if $n=1)$ it turns out that the fractal differential operator from (1.2) is compact, non-negative, and self adjoint operator in $\stackrel{\circ}{W}_{2}^{1}(\Omega)$; its positive eigenvalues $\lambda_{k}(B)$, repeated according to multiplicity and ordered by their magnitude, can be estimated by

$$
\lambda_{k}(B) \sim k^{-\delta} \quad, \quad k \in \mathbb{N},
$$

where $\delta=(2-n+d) / d>0$, see [32, Theorem 30.2] for proofs and details.

Concerning the determination of the eigenvalues, even under the restriction of self similarity for the fractal $\Gamma$, there is no hope to refine (1.4) by an 
asymptotic assertion of type $\lambda_{k}(B)=c k^{-(2-n+d) / d}(1+o(1)), k \rightarrow \infty$, see the discussion in [26] (for $n=1$ ) and [32, Sect. 30.4].

Of course the eigenvalues and eigenfunctions of the operator $B$ can depend on $\Omega$ but, as proved in [32, Theorems 30.2,28.6], it turns out that this is not the case for the exponent $-\delta$ in (1.4).

Motivated by these observations one can replace the inverse of the Dirichlet Laplacian $(-\Delta)^{-1}$ by $(-\Delta+i d)^{-1}$ in $\mathbb{R}^{n}$ or, more generally, by a pseudo-differential operator $b(x, D)$ belonging to some Hörmander class $\Psi_{1, \varrho}^{-\varkappa}\left(\mathbb{R}^{n}\right)$ with $\varkappa>0$ and $0 \leq \varrho \leq 1$, and obtain counterparts of (1.4) for the eigenvalues of the new fractal differential operator, see [32, Sects. 27,28]

New effects appear when $\Gamma$ is replaced by anisotropic $d$-sets in $\mathbb{R}^{2}$ as in [32, Definition 5.2]. In this case instead of (1.4) one has for the eigenvalues of $B$ only two side estimates of type

$$
C_{1} k^{-\omega_{1}} \leq \lambda_{k}(B) \leq C_{2} k^{-\omega_{2}}
$$

for appropriate positive numbers $\omega_{1}$ and $\omega_{2}$ with $\omega_{1} \geq 1 \geq \omega_{2}$, see [32, Theorem 30.7].

However the exponents in (1.5) are not equal in the general case, see the precise formulation in [32, Theorem 30.7]. Some improvements of (1.5) were discussed in [11].

The fact that the two exponents in (1.5) are not equal in the case of general anisotropic $d$-sets should be not a big surprise since one can imagine that the different nature of the (isotropic) operator $-\Delta$ and the (anisotropic) structure of $\Gamma$ would cause difficulties, see also the discussion in [32, Sect. 4.16].

We will consider here only regular anisotropic fractals $\Gamma$ in $\mathbb{R}^{2}$.

The aim of this paper is to show that sharp estimates of type $\lambda_{k} \sim k^{-\delta}$ for the eigenvalues of operators of type $A^{-1} \circ \operatorname{tr}^{\Gamma}$ as in (1.2) can be obtained if there is a certain compatibility between the anisotropy of the fractal $\Gamma$ and the "anisotropy" of the differential operator $A$.

Let $0<d<2$ and let $a=\left(a_{1}, a_{2}\right)$ with $0<a_{2} \leq a_{1}<\infty, a_{1}+a_{2}=$ 2. A regular anisotropic $d$-set with respect to the anisotropy $a$ is, roughly speaking, a compact set $\Gamma \subset \mathbb{R}^{2}$ which can be covered for any $j \in \mathbb{N}_{0}$ with $N_{j} \sim 2^{j d}$ disjoint rectangles having sides parallel to the axes and with side lengths $r_{1}^{j, l}, r_{2}^{j, l}, l=1, \ldots, N_{j}$ satisfying $r_{i}^{j, l} \sim 2^{-j a_{i}}$ for $i=1,2$ (the equivalence constants being independent of $j, l, i)$, cf. [32, Sect. 4.18] and see the precise definition in Sect. 3. Those fractals, usually called Sierpinski carpets in fractal geometry, see for example [19] and [7, Example 9.11], are in fact anisotropic generalisations of the Cantor set in the plane.

Let $\Gamma \subset \mathbb{R}^{2}$ be a regular anisotropic $d$-set with respect to the anisotropy $a=\left(a_{1}, a_{2}\right)$ as described above. Let $t_{1}, t_{2} \in \mathbb{N}$ be such that $t_{1} a_{1}=t_{2} a_{2}=$ 
$t$. Considering the semi - elliptic differential operator $A u(x)=(-1)^{t_{1}} \frac{\partial^{2 t_{1}} u(x)}{\partial x_{1}^{2 t_{1}}}+(-1)^{t_{2}} \frac{\partial^{2 t_{2}} u(x)}{\partial x_{2}^{2 t_{2}}}+u(x), x=\left(x_{1}, x_{2}\right) \in \mathbb{R}^{2}$, and $\operatorname{tr}^{\Gamma}$ the trace operator in the interpretation (1.1), the main objective of our work is to study spectral properties of the operator $A^{-1} \circ \operatorname{tr}^{\Gamma}$ acting in the anisotropic Sobolev space $W^{\left(t_{1}, t_{2}\right)}\left(\mathbb{R}^{2}\right)$.

We will show that $A^{-1} \circ \operatorname{tr}^{\Gamma}$ is compact, non-negative, and self adjoint in $W^{\left(t_{1}, t_{2}\right)}\left(\mathbb{R}^{2}\right)$ and that there exist constants $c, C>0$ such that its positive eigenvalues, repeated according to multiplicity and ordered by their magnitude, can be estimated by

$$
c k^{-\frac{1}{d}(d+2 t-2)} \leq \lambda_{k}\left(A^{-1} \circ \operatorname{tr}^{\Gamma}\right) \leq C k^{-\frac{1}{d}(d+2 t-2)} \quad, \quad k \in \mathbb{N} .
$$

The precise formulation of (1.6) is contained in Theorem 4.1.

We think that the proof of Theorem 4.1, in particular of the proof of the estimates (1.6), is of interest for its own sake.

First it is proved that the $L_{p}$ - spaces on the regular anisotropic fractal $\Gamma$ can be identified with certain subspaces of some anisotropic Besov spaces, see the precise formulations in Sect. 3.

The results in Sect. 3 are not only anisotropic counterparts of the results obtained in [33] (where H. Triebel and H. Winkelvoß proved that there is a perfect link between $L_{p^{-}}$spaces on isotropic $d$-sets and the Fourier analytically defined - isotropic - Besov spaces $B_{p q}^{s}$ on $\mathbb{R}^{n}$, see also [32, Theorems 18.2,18.6]), but they play a key role in the proof of the main result of this paper, see Theorem 4.1 and the proof in Sect. 6.

Secondly, the methods how the two sides of (1.6) are obtained are rather different.

Using the subatomic decomposition theorem for anisotropic function spaces from [10] we estimate the entropy numbers of some embeddings between function spaces. These estimates generalise to the anisotropic case Theorems 20.5, 20.6 from [32] and complement Theorem 22.2 in [32]; to return to the eigenvalues and to the proof of the estimate from above in (1.6) one has then only to apply Carl's inequality (see [2]).

To prove the estimate from below in (1.6) we investigate the approximation numbers of the operator $\left(A^{-1} \circ \operatorname{tr}^{\Gamma}\right)^{1 / 2}$ and here we use the atomic decomposition theorem in anisotropic function spaces proved in [10].

Briefly about the organising of the paper.

We start (in Sect. 2) with a collection of basic facts concerning anisotropic Besov and Sobolev spaces. Regular anisotropic $d$-sets and $L_{p}$ - spaces on such fractals are presented in Sect. 3. The main result is formulated in Sect. 4. Proofs are given in Sects. 5 and 6. 
As usual $\mathbb{N}$ are the natural numbers and $\mathbb{N}_{0}=\mathbb{N} \cup\{0\}$. For a normed or quasi-normed space $X$ we denote by $\|x \mid X\|$ the norm of the vector $x$. The embedding of the quasi-normed space $X$ into the quasi-normed space $Y$ is denoted $X \hookrightarrow Y$.

All unimportant positive constants are denoted with $c$, occasionally with additional subscripts within the same formulas.

\section{Basic facts on anisotropic function spaces}

If $1<p<\infty$ and $\left(s_{1}, s_{2}\right)$ is a pair of natural numbers then the classical anisotropic Sobolev space $W_{p}^{\left(s_{1}, s_{2}\right)}\left(\mathbb{R}^{2}\right)$ is the collection of all tempered distributions $f \in S^{\prime}\left(\mathbb{R}^{2}\right)$ such that

$$
\left\|f\left|W_{p}^{\left(s_{1}, s_{2}\right)}\left(\mathbb{R}^{2}\right)\|=\| f\right| L_{p}\left(\mathbb{R}^{2}\right)\right\|+\left\|\frac{\partial^{s_{1}} f}{\partial x_{1}^{s_{1}}}\left|L_{p}\left(\mathbb{R}^{2}\right)\|+\| \frac{\partial^{s_{2}} f}{\partial x_{2}^{s_{2}}}\right| L_{p}\left(\mathbb{R}^{2}\right)\right\|
$$

is finite. In contrast to the usual (isotropic) Sobolev space $\left(s_{1}=s_{2}\right)$ the smoothness properties of an element from $W_{p}^{\left(s_{1}, s_{2}\right)}\left(\mathbb{R}^{2}\right)$ depend on the chosen direction in $\mathbb{R}^{2}$.

The number $s$ defined by

$$
\frac{1}{s}=\frac{1}{2}\left(\frac{1}{s_{1}}+\frac{1}{s_{2}}\right)
$$

is usually called the "mean smoothness" and $a=\left(a_{1}, a_{2}\right)$, where $a_{1}=\frac{s}{s_{1}}$, $a_{2}=\frac{s}{s_{2}}$, characterises the anisotropy. Sometimes we will use the notation $W_{p}^{s, a}\left(\mathbb{R}^{2}\right)$ for these spaces.

A pair $a=\left(a_{1}, a_{2}\right)$ with $0<a_{1}, a_{2}<\infty$ and $a_{1}+a_{2}=2$ is called an anisotropy.

If $a=(1,1)$ then we speak about the "isotropic case".

Let $1<p<\infty, s \in \mathbb{R}$ and $s_{1}=\frac{s}{a_{1}}$ and $s_{2}=\frac{s}{a_{2}}$.

Anisotropic Bessel potential spaces, or fractional Sobolev spaces, defined by

$$
\begin{aligned}
& H_{p}^{s, a}\left(\mathbb{R}^{2}\right) \\
& =\left\{f \in S^{\prime}\left(\mathbb{R}^{2}\right):\left\|\left(\left(\left(1+\xi_{1}^{2}\right)^{\frac{s_{1}}{2}}+\left(1+\xi_{2}^{2}\right)^{\frac{s_{2}}{2}}\right) \hat{f}\right)^{\vee} \mid L_{p}\left(\mathbb{R}^{2}\right)\right\|<\infty\right\}
\end{aligned}
$$

generalise in a natural way the above spaces (as usual, $\widehat{f}, \check{f}$ are respectively the Fourier and the inverse Fourier transform of $f \in S^{\prime}\left(\mathbb{R}^{2}\right)$ ).

We recall now the definition of anisotropic Besov spaces on $\mathbb{R}^{2}$. 
First we fix some notation. The action of $\theta \in[0, \infty)$ on $x=\left(x_{1}, x_{2}\right) \in$ $\mathbb{R}^{2}$ is defined by the formula:

$$
\theta^{a} x=\left(\theta^{a_{1}} x_{1}, \theta^{a_{2}} x_{2}\right) .
$$

For $\theta>0$ and $s \in \mathbb{R}$ let $\theta^{s a} x=\left(\theta^{s}\right)^{a} x$. In particular let $\theta^{-a} x=\left(\theta^{-1}\right)^{a} x$ and $2^{-j a} x=\left(2^{-j}\right)^{a} x$.

For $x=\left(x_{1}, x_{2}\right) \in \mathbb{R}^{2}, x \neq 0$, let $|x|_{a}$ be the unique positive number $\theta$ such that

$$
\frac{x_{1}^{2}}{\theta^{2 a_{1}}}+\frac{x_{2}^{2}}{\theta^{2 a_{2}}}=1
$$

and let $|0|_{a}=0$. By M. Yamazaki, [35, Theorem 1.4/3,8], $|\cdot|{ }_{a}$ is an anisotropic distance function in $C^{\infty}\left(\mathbb{R}^{2} \backslash\{0\}\right)$. Remark that in the isotropic case $|x|_{a}$ is the Euclidean distance of $x$ to the origin.

Let $\varphi_{0}$ a $C^{\infty}$ function on $\mathbb{R}^{2}, \varphi_{0}(x)=1$ if $|x|_{a} \leq 1$, supp $\varphi_{0} \subset\{x \in$ $\left.\mathbb{R}^{2}:|x|_{a} \leq 2\right\}$ and $\varphi_{j}(x)=\varphi_{0}\left(2^{-j a} x\right)-\varphi_{0}\left(2^{(-j+1) a} x\right)$ if $j \in \mathbb{N}$. Then $\sum_{j=0}^{\infty} \varphi_{j}(x)=1$ if $x \in \mathbb{R}^{2}$ and $\left(\varphi_{j}\right)_{j \in \mathbb{N}_{0}}$ is a smooth anisotropic dyadic resolution of unity, cf. [24, Sect. 4.2].

Let $0<p \leq \infty, 0<q \leq \infty, s \in \mathbb{R}$. The anisotropic Besov space $B_{p q}^{s, a}\left(\mathbb{R}^{2}\right)$ consists of all tempered distributions $f \in S^{\prime}\left(\mathbb{R}^{2}\right)$ for which the quasi-norm

$$
\left\|f \mid B_{p q}^{s, a}\left(\mathbb{R}^{2}\right)\right\|=\left(\sum_{j=0}^{\infty} 2^{j s q}\left\|\left(\varphi_{j} \widehat{f}\right)^{\vee} \mid L_{p}\left(\mathbb{R}^{2}\right)\right\|^{q}\right)^{1 / q}
$$

(with usual modification if $q=\infty$ ) is finite.

These are quasi-Banach spaces (Banach spaces if $p \geq 1$ and $q \geq 1$ ) which are independent of the choice of $\left(\varphi_{j}\right)_{j \in \mathbb{N}_{0}}$.

The space $H_{2}^{s, a}\left(\mathbb{R}^{2}\right)=B_{22}^{s, a}\left(\mathbb{R}^{2}\right)$ is the anisotropic fractional Sobolev space. In particular, if $s_{1}=\frac{s}{a_{1}}$ and $s_{2}=\frac{s}{a_{2}}$ are natural numbers then $B_{22}^{s, a}\left(\mathbb{R}^{2}\right)=W_{2}^{s, a}\left(\mathbb{R}^{2}\right)$.

Directly from the definition we have

$$
B_{p q_{0}}^{s+\varepsilon, a}\left(\mathbb{R}^{2}\right) \hookrightarrow B_{p q_{1}}^{s, a}\left(\mathbb{R}^{2}\right) \quad \text { if } \quad \varepsilon>0,
$$

for any $s \in \mathbb{R}$, any $0<p \leq \infty$ and any $0<q_{0}, q_{1} \leq \infty$ see [28, Proposition 2.3.2/2]. Anisotropic function spaces of $F_{p q}^{s, a}\left(\mathbb{R}^{2}\right)$ type, $0<p<\infty$, are defined changing the roles of the spaces $L_{p}\left(\mathbb{R}^{2}\right)$ and $l_{q}$ in the above definition but we do not stress this point here.

The above spaces are denoted $B_{p q}^{s}\left(\mathbb{R}^{2}\right)$ in the isotropic case. A systematic treatment of the theory of isotropic $B_{p q}^{s}\left(\mathbb{R}^{2}\right)$ and $F_{p q}^{s}\left(\mathbb{R}^{2}\right)$ spaces may be found in the books of $\mathrm{H}$. Triebel [28], [30]. 


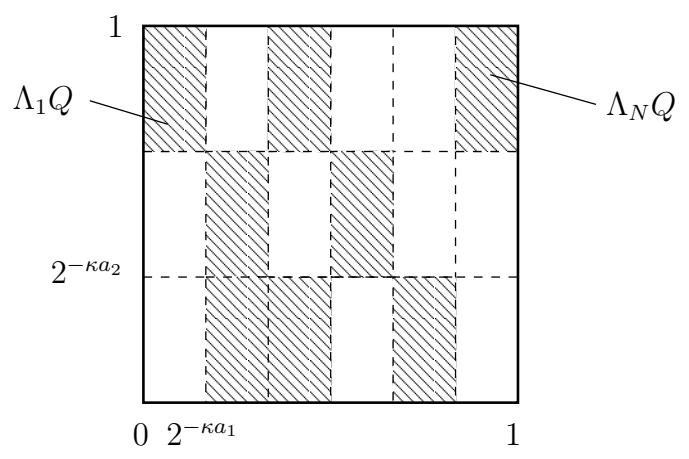

Fig. 1.

Anisotropic function spaces have been studied in great detail by S. M. Nikol'skij, see [22], and by O. V. Besov, V. P. Il'in and S. M. Nikol'skij, see [1]. In [10] we have given a longer list of contributions to the theory of anisotropic function spaces.

\section{$3 L_{p}$ - spaces on anisotropic Cantor sets}

\subsection{Regular anisotropic d-sets in $\mathbb{R}^{2}$}

Example 3.1 The Sierpinski carpet. Let $Q=[0,1] \times[0,1]$ and let $\log$ be taken with respect to the base 2 , let $1<K_{2} \leq K_{1}$ be natural numbers and let

$$
\kappa=\frac{1}{2} \log \left(K_{1} K_{2}\right) \quad, \quad a_{1}=\frac{\log K_{1}}{\kappa} \quad, \quad a_{2}=\frac{\log K_{2}}{\kappa} .
$$

In particular $0<a_{2} \leq a_{1}<\infty, a_{1}+a_{2}=2$, and $a=\left(a_{1}, a_{2}\right)$ is an anisotropy.

Let $\left(\Lambda_{m}\right)_{m=1}^{N}$ be $N \geq 2$ contractions of $\mathbb{R}^{2}$ into itself specified by

$$
\Lambda_{m} \quad: \quad x=\left(x_{1}, x_{2}\right) \mapsto\left(\eta_{1}^{m} 2^{-\kappa a_{1}} x_{1}, \eta_{2}^{m} 2^{-\kappa a_{2}} x_{2}\right)+x^{m}
$$

for every $m=1, \ldots, N$ where $\eta_{1}^{m}, \eta_{2}^{m} \in\{-1,+1\}$ (including possible reflections). We assume $\Lambda_{m} Q \subset Q$ for all $m=1, \ldots, N, \Lambda_{m} \stackrel{\circ}{Q} \cap \Lambda_{m^{\prime}} \stackrel{\circ}{Q}=$ $\emptyset$ if $m \neq m^{\prime}$ and $\sum_{m=1}^{N} \operatorname{vol} \Lambda_{m} Q<1$. For example, we suppose that the rectangles $\Lambda_{m} Q$ are located in the columns as indicated in Fig. 1.

Let

$$
\Lambda Q=(\Lambda Q)^{1}=\bigcup_{m=1}^{N} \Lambda_{m} Q \quad ; \quad(\Lambda Q)^{0}=Q \quad ;
$$




$$
(\Lambda Q)^{\nu}=\Lambda\left((\Lambda Q)^{\nu-1}\right)=\bigcup_{1 \leq m_{1}, \ldots, m_{\nu} \leq N} \Lambda_{m_{1}} \circ \ldots \circ \Lambda_{m_{\nu}} Q \quad ; \quad \nu \in \mathbb{N} .
$$

This sequence of sets is monotonically decreasing and by [6, Theorem 8.3] its limit

$$
\Gamma=(\Lambda Q)^{\infty}=\bigcap_{\nu \in \mathbb{N}}(\Lambda Q)^{\nu}=\lim _{\nu \rightarrow \infty}(\Lambda Q)^{\nu}
$$

is the uniquely determined fractal generated by the contractions $\left(\Lambda_{m}\right)_{m=1}^{N}$.

Fractals constructed in this way are anisotropic generalisations of the Cantor set in $\mathbb{R}^{2}$ and were called Sierpinski carpets in [19] or regular anisotropic fractals in [32, Sect. 4.18].

The Hausdorff dimension for this type of fractals was computed in [19], see also [7, Example 9.11]. We want to remark that it depends not only on the number of rectangles selected at each stage but also on their relative position, more precisely it depends on $K_{1}, K_{2}$ and on the number of rectangles $\Lambda_{m} Q$ selected in each column.

Let $\left(\Lambda_{m}\right)_{m=1}^{N}$ be the $N \geq 2$ affine maps introduced in (3.2). The affine dimension of $\Gamma=(\Lambda Q)^{\infty}$, see [32, Definition 4.12], is the uniquely determined positive number $d=\operatorname{dim}_{A} \Gamma$ such that

$$
\sum_{m=1}^{N}\left(\operatorname{vol} \Lambda_{m} Q\right)^{d / 2}=1 .
$$

In what follows we give the definition of regular anisotropic $d$-sets and after that we will show that Sierpinski carpets are typical examples of those sets.

If $j \in \mathbb{N}_{0}$ and $N_{j} \in \mathbb{N}_{0}$ we deal with sets of open rectangles $\left\{R_{j l}\right.$ : $\left.l=1, \ldots, N_{j}\right\}$ in $\mathbb{R}^{2}$ having sides parallel to the axes; the side length of the rectangle $R_{j l}$ with respect to the $x_{i}$ - axis is denoted by $r_{i}^{j, l}$ where $i=1,2$. We will always assume that the side lengths of the rectangles $R_{j l}$ are ordered in the same way, for example $r_{1}^{j, l} \leq r_{2}^{j, l}$ for any $j \in \mathbb{N}_{0}$ and any $l=1, \ldots, N_{j}$.

Definition 3.2 Let $Q$ be a cube in $\mathbb{R}^{2}$ with side length 1 , let $0<d<2$, let $a=\left(a_{1}, a_{2}\right)$ a given anisotropy and let $c_{1}, c_{2}>0$ given numbers.

Let $N_{0}=1$ and for any $j \in \mathbb{N}$ let $N_{j}$ be a natural number satisfying

$$
c_{1} 2^{j d} \leq N_{j} \leq c_{2} 2^{j d} .
$$

A compact set $\Gamma \subset \mathbb{R}^{2}$ is called a regular anisotropic d-set (with respect to the anisotropy a) if for any $j \in \mathbb{N}_{0}$ there exists a finite sequence of open rectangles $\left\{R_{j l}: l=1, \ldots, N_{j}\right\}$ having sides parallel to the axes, $R_{01}=\stackrel{\circ}{Q}$, such that: 
(i) there exists a constant $0<c_{0} \leq 1$ such that for all $j \in \mathbb{N}_{0}$ and all $l=1, \ldots, N_{j}$

$$
\left(c_{0} 2^{-j}\right)^{a_{i}} \leq r_{i}^{j, l} \leq 2^{-j a_{i}} \quad, \quad i=1,2 ;
$$

(ii) if $l \neq l^{\prime}$ then $R_{j l} \cap R_{j l^{\prime}}=\emptyset$;

(iii) for any rectangle $R_{j+1, k}$ there exists a rectangle $R_{j l}, l=l(k)$, such that $R_{j+1, k} \subset R_{j l}$;

(iv) for any $j \in \mathbb{N}_{0}$ and any $l=1, \ldots, N_{j}$

$$
\left(\operatorname{vol} R_{j l}\right)^{\frac{d}{2}}=\sum_{R_{j+1, k} \subset R_{j l}}\left(\operatorname{vol} R_{j+1, k}\right)^{\frac{d}{2}} ;
$$

(v) $\Gamma=\bigcap_{j=0}^{\infty} \bigcup_{l=1}^{N_{j}} \overline{R_{j l}}$.

Remark 3.3 If there exists a number $0<c \leq 1$ such that $c 2^{-2 j} \leq \operatorname{vol} R_{j l} \leq$ $2^{-2 j}$ for any $j \in \mathbb{N}_{0}$ and any $l=1, \ldots, N_{j}$, and if condition (i) in the above definition is replaced by:

(i*) there exists a number $0 \leq \varepsilon \leq 1$ (called the anisotropic deviation) and a number $j_{0} \in \mathbb{N}_{0}$ such that the side lengths $r_{1}^{j, l}$ and $r_{2}^{j, l}$ of the rectangle $R_{j l}$ satisfy

$$
\begin{aligned}
& 2^{-j(1+\varepsilon)} \leq r_{1}^{j, l} \leq r_{2}^{j, l} \leq 2^{-j(1-\varepsilon)} \\
& \text { for any } \quad j \geq j_{0} \quad \text { and any } \quad l=1, \ldots, N_{j}
\end{aligned}
$$

then we obtain the definition of an anisotropic $d$-set (with anisotropic deviation $\varepsilon$ ) as it was given by $\mathrm{H}$. Triebel in [32, 5.2].

Theorem 3.4 Let $a_{1}, a_{2}$ defined in (3.1) and let $\Gamma$ be the Sierpinski carpet generated by the contractions (3.2).

If $\Gamma$ has affine dimension $d$ according to (3.4) then $\Gamma$ is a regular anisotropic d-set with respect to the anisotropy $a=\left(a_{1}, a_{2}\right)$.

Proof. By construction we have $0<d<2$ and $N=2^{\kappa d}$. It is clear that for any $\nu \in \mathbb{N}$ and any $m_{1}, \ldots, m_{\nu} \in\{1, \ldots, N\}$ the set $\Lambda_{m_{1}} \circ \ldots \circ \Lambda_{m_{\nu}} Q$ is a rectangle having sides parallel to the axes and having side lengths $2^{-\nu \kappa a_{1}}$, $2^{-\nu \kappa a_{2}}$; the number of such rectangles is $N^{\nu}=2^{\nu \kappa d}$.

$$
\begin{aligned}
& \text { If } j \in \mathbb{N} \text { let } \nu=\nu(j)=\left[\frac{j}{\kappa}\right]+1 \text {, let } N_{j}=N^{\nu(j)} \text { and let } \\
& \left\{R_{j l}: l=1, \ldots, N_{j}\right\}=\left\{\Lambda_{m_{1}} \circ \ldots \circ \Lambda_{m_{\nu}} \stackrel{\circ}{Q}: 1 \leq m_{1}, \ldots, m_{\nu} \leq N\right\} .
\end{aligned}
$$




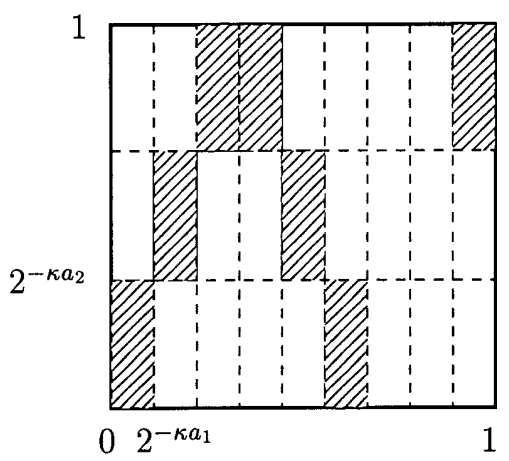

Fig. 2.

Clearly we find two constants $c_{1}, c_{2}>0$ such that $c_{1} 2^{j d} \leq N_{j} \leq c_{2} 2^{j d}$ for any $j \in \mathbb{N}$. Furthermore, the side lengths $r_{1}^{j, l}$ and $r_{2}^{j, l}$ of the rectangle $R_{j l}$ satisfy $r_{1}^{j, l} \leq r_{2}^{j, l}$ and

$$
\left(c_{0} 2^{-j}\right)^{a_{i}} \leq r_{i}^{j, l} \leq 2^{-j a_{i}} \quad \text { with } \quad i=1,2,
$$

where $c_{0}=2^{-\kappa}$. Using (3.4) it is easy to see that all the other required properties are satisfied.

Theorem 3.5 ([32, Theorems 5.5,4.15]) Let $\Gamma$ be a regular anisotropic $d$-set with respect to the anisotropy $a=\left(a_{1}, a_{2}\right)$ and let $\left\{R_{j l}: j \in \mathbb{N}_{0}, l=\right.$ $\left.1, \ldots, N_{j}\right\}$ be the rectangles from Definition 3.2. Then there exists a Radon measure $\mu$ in $\mathbb{R}^{2}$ uniquely determined with supp $\mu=\Gamma$ and

$$
\mu\left(\Gamma \cap R_{j l}\right)=\left(\operatorname{vol} R_{j l}\right)^{d / 2} \quad \text { for all } \quad j \in \mathbb{N}_{0} \quad \text { and all } \quad l=1, \ldots, N_{j} .
$$

Example 3.6 In specification of the situation in Fig. 1 we suppose now that in each column precisely one rectangle $\Lambda_{m} Q$ is located. Let $\frac{K_{1}}{K_{2}}=2 k+1$ for some $k \in \mathbb{N}$ and let the rectangles $\Lambda_{m} Q$ be arranged as depicted in Fig. 2: we choose in (3.2) always $\eta_{2}^{m}=1$ and we choose $\eta_{1}^{m}=1$ in the first $K_{2}$ columns, $\eta_{1}^{m}=-1$ in the second $K_{2}$ columns (additional reflection) then again $\eta_{1}^{m}=1$ in the third $K_{2}$ columns and so on, see Fig. 2 .

Under these assumptions the resulting Sierpinski carpet fractal $\Gamma$ is the graph of a continuous function, for a proof see [32, Sect. 4.21]. It is clear that $\Gamma$ may be interpreted as a generalisation of Hironaka's curve (briefly presented in [19]).

Moreover, $\Gamma$ is an isotropic $D$-set, see (1.3), where $D=\operatorname{dim}_{H} \Gamma=$ $2-a_{2} / a_{1}$, see [32, Sects. 4.22, 4.23]. It is not difficult to see that $\Gamma$ is a regular anisotropic $d$-set with $d=a_{1}$. 
Comments. As remarked in [32, Sects. 4.14,4.22] if the anisotropy $a=$ $\left(a_{1}, a_{2}\right)$ is non-trivial, that means $a=\left(a_{1}, a_{2}\right) \neq(1,1)$, then the number $d$ from (3.4) and the Hausdorff dimension $\operatorname{dim}_{H} \Gamma$ (computed in [19]) of the Sierpinski carpet $\Gamma$, see (3.3), generated by the contractions defined in (3.2), are completely unrelated.

Furthermore, Example 3.6 shows that it can happen that the regular anisotropic $d$-set $\Gamma$ is also an isotropic $d^{\prime}$-set with $d \neq d^{\prime}$.

From (1.3) we know that if $\Gamma$ is an isotropic $d$-set with underlying measure $\mu$ and if $0<\varkappa<1$ then

$$
\mu(B(\gamma, \varkappa r) \cap \Gamma) \sim \mu(B(\gamma, r) \cap \Gamma) \sim r^{d}
$$

where the equivalence constants depend on $\varkappa$ but not on $\gamma \in \Gamma$ and $0<$ $r \leq 1$.

For a regular anisotropic $d$-set $\Gamma$ we have (3.5) but no counterpart of (3.6). At least a weak version of (3.6) will be needed. If $0<\varkappa<1$ then $\varkappa R_{j l}$ denotes the rectangle concentric with $R_{j l}$ and with side lengths respectively $\varkappa r_{1}^{j, l}, \varkappa r_{2}^{j, l}$.

Definition 3.7 A regular anisotropic d-set equipped with measure $\mu$ according to Theorem 3.5 is called proper if there exist two numbers $0<\varkappa<1$ and $0<c \leq 1$ such that

$$
\mu\left(\Gamma \cap \varkappa R_{j l}\right) \geq c\left(\operatorname{vol} R_{j l}\right)^{d / 2} \quad, \quad j \in \mathbb{N}_{0} \quad, \quad l=1, \ldots, N_{j},
$$

where $\left\{R_{j l}: j \in \mathbb{N}_{0}, l=1, \ldots, N_{j}\right\}$ are the rectangles from Definition 3.2.

Remark 3.8 If $\Gamma$ is a Sierpinski carpet generated by the linear contractions (3.2), following the lines of the proof of [32, Proposition 5.13] it turns out that if $\Gamma \cap \stackrel{\circ}{Q} \neq \emptyset$ then $\Gamma$ is proper. Hence condition (3.7) is rather natural.

\subsection{Embeddings in anisotropic Besov spaces}

Let $\Gamma$ be a regular anisotropic $d$-set with respect to the given anisotropy $a=\left(a_{1}, a_{2}\right)$. The $L_{p^{-}}$spaces on $\Gamma, 0<p \leq \infty$, are introduced in the usual way with respect to the underlying Radon measure $\mu$ on $\Gamma$ according to Theorem 3.5 .

If $1 \leq p \leq \infty$ any $f_{\Gamma} \in L_{p}(\Gamma)$ will be interpreted as a tempered distribution $f \in S^{\prime}\left(\mathbb{R}^{2}\right)$ given by:

$$
f(\varphi)=\int_{\Gamma} f_{\Gamma}(\gamma)(\varphi \mid \Gamma)(\gamma) d \mu(\gamma), \quad \varphi \in S\left(\mathbb{R}^{2}\right),
$$

where $\varphi \mid \Gamma$ is the restriction of $\varphi$ to $\Gamma$. 
Theorem 3.9 Let $0<d<2$ and let $\Gamma$ be a regular anisotropic $d$-set in $\mathbb{R}^{2}$ with respect to the anisotropy $a=\left(a_{1}, a_{2}\right)$. If $1<p \leq \infty$ and $\frac{1}{p}+\frac{1}{p^{\prime}}=1$ then (in the sense of (3.8))

$$
L_{p}(\Gamma)=\left\{f \in B_{p \infty}^{-\frac{2-d}{p^{\prime}}, a}\left(\mathbb{R}^{2}\right): f(\varphi)=0 \quad \text { if } \quad \varphi \in S\left(\mathbb{R}^{2}\right), \varphi \mid \Gamma=0\right\} .
$$

We will restrict ourselves in Sect. 5.1 to the proof of the embedding " $\hookrightarrow$ " because only this part of the theorem will be used in the main result of this work. The reverse embedding can be proved using the technique from [33] (adapted to our anisotropic situation); for details one can see [8, Theorem 3.11].

\subsection{Traces}

Assume that $\Gamma$ is a regular anisotropic $d$-set in $\mathbb{R}^{2}$ with respect to the anisotropy $a=\left(a_{1}, a_{2}\right)$. If $\varphi \in S\left(\mathbb{R}^{2}\right)$ then $\operatorname{tr}_{\Gamma} \varphi=\varphi \mid \Gamma$ makes sense pointwise. If $0<p, q<\infty$ and $s \in \mathbb{R}$ then the embedding $\operatorname{tr}_{\Gamma} B_{p q}^{s, a}\left(\mathbb{R}^{2}\right) \hookrightarrow$ $L_{p}(\Gamma)$ must be understood as follows: there exists a positive number $c>0$ such that for any $\varphi \in S\left(\mathbb{R}^{2}\right): \quad\left\|\operatorname{tr}_{\Gamma} \varphi\left|L_{p}(\Gamma)\|\leq c\| \varphi\right| B_{p q}^{s, a}\left(\mathbb{R}^{2}\right)\right\|$.

Since $S\left(\mathbb{R}^{2}\right)$ is dense in $B_{p q}^{s, a}\left(\mathbb{R}^{2}\right)$ for $0<p, q<\infty$ this inequality can be extended by completion to any $f \in B_{p q}^{s, a}\left(\mathbb{R}^{2}\right)$ and the resulting function is denoted $\operatorname{tr}_{\Gamma} f$.

In addition, the equality $\operatorname{tr}_{\Gamma} B_{p q}^{s, a}\left(\mathbb{R}^{2}\right)=L_{p}(\Gamma)$ means that any $f_{\Gamma} \in$ $L_{p}(\Gamma)$ is the trace of a suitable $g \in B_{p q}^{s, a}\left(\mathbb{R}^{2}\right)$ on $\Gamma$ and $\left\|f_{\Gamma} \mid L_{p}(\Gamma)\right\| \sim$ $\inf \left\{\left\|g \mid B_{p q}^{s, a}\left(\mathbb{R}^{2}\right)\right\|: \operatorname{tr}_{\Gamma} g=f_{\Gamma}\right\}$.

Theorem 3.10 Let $0<d<2$ and let $\Gamma$ be a regular anisotropic $d$-set in $\mathbb{R}^{2}$ with respect to the anisotropy $a=\left(a_{1}, a_{2}\right)$. If $\frac{d}{2}<p<\infty$ and $0<q \leq \min (1, p)$ then

$$
\operatorname{tr}_{\Gamma} B_{p q}^{\frac{2-d}{p}, a}\left(\mathbb{R}^{2}\right)=L_{p}(\Gamma)
$$

We want to remark that in Sect. 5.2 we will prove only the embedding $\operatorname{tr}_{\Gamma} B_{p q}^{\frac{2-d}{p}, a}\left(\mathbb{R}^{2}\right) \hookrightarrow L_{p}(\Gamma)$. Only this part of Theorem 3.10 will be used in the main result of this paper. The reverse embedding can be proved using the technique from [33], see also [8, Theorem 3.12].

As mentioned in the Introduction, Theorems 3.9 and 3.10 pave the way to the main result of this work but they seem to be of independent interest since they are anisotropic counterparts of Theorems 2 and 3 in [33], see also [32, Theorems 18.2,18.6]. The extension of Theorem 3.10 to all $0<p<\infty$ and the obtaining of a counterpart of Theorem 18.12 in [32] were discussed in [8, Sect. 4.3]. 


\section{The main result}

Let $\Gamma$ be a regular anisotropic $d$-set with respect to the anisotropy $a=$ $\left(a_{1}, a_{2}\right)$.

In the sequel we shall not distinguish between $f_{\Gamma}$ as an element of some $L_{p}(\Gamma)$ and as the distribution $f$ belonging to some $B_{p \infty}^{-s, a}\left(\mathbb{R}^{2}\right)$ according to (3.9) and (3.8).

To avoid any misunderstanding we emphasise that the trace operator has two different meanings which we distinguish by $\operatorname{tr}_{\Gamma}$ and $\operatorname{tr}^{\Gamma}$ if extra clarity is desirable. Let, for example, $1<p<\infty$, then by (3.10)

$$
\operatorname{tr}_{\Gamma}: B_{p 1}^{\frac{2-d}{p}, a}\left(\mathbb{R}^{2}\right) \rightarrow L_{p}(\Gamma)
$$

and if one applies in addition (3.9)

$$
\operatorname{tr} \Gamma: B_{p 1}^{\frac{2-d}{p}, a}\left(\mathbb{R}^{2}\right) \rightarrow B_{p \infty^{-\frac{2-d}{p^{\prime}}, a}}\left(\mathbb{R}^{2}\right) .
$$

The latter can be rephrased asking for an optimal extension of $\operatorname{tr}^{\Gamma}$ considered as a mapping from $D\left(\mathbb{R}^{2}\right)$ into $D^{\prime}\left(\mathbb{R}^{2}\right)$ given by (1.1).

Let $t_{1}, t_{2} \in \mathbb{N}$ and let $t \in \mathbb{R}$ defined from

$$
\frac{1}{t}=\frac{1}{2}\left(\frac{1}{t_{1}}+\frac{1}{t_{2}}\right) \text {. }
$$

Let $A$ be the operator defined by

$$
A u(x)=(-1)^{t_{1}} \frac{\partial^{2 t_{1}} u(x)}{\partial x_{1}^{2 t_{1}}}+(-1)^{t_{2}} \frac{\partial^{2 t_{2}} u(x)}{\partial x_{2}^{2 t_{2}}}+u(x)
$$

where $x=\left(x_{1}, x_{2}\right) \in \mathbb{R}^{2}$. Using elementary properties of the Fourier transform we have

$$
A u=\left(\left(1+\xi_{1}^{2 t_{1}}+\xi_{2}^{2 t_{2}}\right) \widehat{u}\right)^{\vee}
$$

for any $u \in S^{\prime}\left(\mathbb{R}^{2}\right)$.

It is well known, see for example [18], or it is easy to prove, that $A$ is a lift operator for the scale $B_{p q}^{s, a}\left(\mathbb{R}^{2}\right), s \in \mathbb{R}, 0<p \leq \infty, 0<q \leq \infty$. More precisely, $A$ maps any space $B_{p q}^{s, a}\left(\mathbb{R}^{2}\right)$ isomorphic onto $B_{p q}^{s-2 t, a}\left(\mathbb{R}^{2}\right)$ and $\left\|A(\cdot) \mid B_{p q}^{s-2 t, a}\left(\mathbb{R}^{2}\right)\right\|$ is an equivalent quasi-norm on $B_{p q}^{s-2 t, a}\left(\mathbb{R}^{2}\right)$.

The inverse $A^{-1}$ of $A$ has to be understood in this way. 
Theorem 4.1 Let $0<d<2$ and let $\Gamma \subset \Omega$ be a regular anisotropic $d$-set with respect to the anisotropy $a=\left(a_{1}, a_{2}\right)$. Let tr ${ }^{\Gamma}$ be the trace operator in the interpretation (4.2) and (1.1) whereas $t_{\Gamma}$ stands for the trace operator according to (4.1).

Let $t \in \mathbb{R}$ such that $t_{1}=\frac{t}{a_{1}} \in \mathbb{N}$ and $t_{2}=\frac{t}{a_{2}} \in \mathbb{N}$ and let $A$ be the operator from (4.3).

(i) The operator

$$
T=A^{-1} \circ \operatorname{tr}^{\Gamma}
$$

is compact, non-negative, self adjoint in $W_{2}^{\left(t_{1}, t_{2}\right)}\left(\mathbb{R}^{2}\right)$ and has null space

$$
N(T)=\left\{f \in W_{2}^{\left(t_{1}, t_{2}\right)}\left(\mathbb{R}^{2}\right): \operatorname{tr}_{\Gamma} f=0\right\} .
$$

Furthermore, $T$ is generated by the quadratic form in $W_{2}^{\left(t_{1}, t_{2}\right)}\left(\mathbb{R}^{2}\right)$

$$
\int_{\Gamma} f(\gamma) \overline{g(\gamma)} d \mu(\gamma)=(T f, g)_{W_{2}^{\left(t_{1}, t_{2}\right)}\left(\mathbb{R}^{2}\right)} \text { where } f, g \in W_{2}^{\left(t_{1}, t_{2}\right)}\left(\mathbb{R}^{2}\right) \text {, }
$$

and $\mu$ is the Radon measure according to Theorem 3.5.

(ii) There exists a constant $C>0$ such that the positive eigenvalues $\lambda_{k}(T)$ of $T$, repeated according to multiplicity and ordered by their magnitude, can be estimated by

$$
\lambda_{k}(T) \leq C k^{-\frac{1}{d}(d+2 t-2)} \quad, \quad k \in \mathbb{N} .
$$

If, in addition, $\Gamma$ is proper according to Definition 3.7 then there exists a constant $c>0$ such that

$$
c k^{-\frac{1}{d}(d+2 t-2)} \leq \lambda_{k}(T) \quad, \quad k \in \mathbb{N} .
$$

The main ideas of the proof of Theorem 4.1 were briefly mentioned in the Introduction. The detailed proof is given in Sect. 6.

Remark 4.2 The additional assumption on $\Gamma$ to be proper in the estimate (4.8) excludes by Remark 3.8 only pathological cases where the whole fractal retreats in the boundary of the starting square.

Remark 4.3 It is clear how one can define $n$-dimensional $(n \geq 2)$ regular anisotropic $d$-sets, with $n$-dimensional Sierpinski carpets as standard examples. It will turn out that the proof of the above theorem can be done in an $n$-dimensional setting using the same arguments. Considering the $n$ dimensional counterpart of the operator $A$ the estimates for the eigenvalues would be (if $n-2 t<d<n$ )

$$
c k^{-\frac{1}{d}(d+2 t-n)} \leq \lambda_{k}(T) \leq C k^{-\frac{1}{d}(d+2 t-n)} \quad, \quad k \in \mathbb{N},
$$

and this is in accord with [32, Theorem 28.6]. 
Comments. Some interesting results can be obtained by specialising $t_{1}$ and $t_{2}$.

(i) If $t_{1}=t_{2}=1$ then $A=-\Delta+i d$ and $\Gamma$ is an isotropic $d$-set (in particular the Sierpinski carpet would be generated by the similarities $\left(\Lambda_{m}\right)_{m=1}^{N}$ ). Consequently,

$$
c k^{-1} \leq \lambda_{k}\left((-\Delta+i d)^{-1} \circ t r^{\Gamma}\right) \leq C k^{-1} \quad, \quad k \in \mathbb{N} .
$$

(ii) If $t_{1}=1$ and $t_{2}=2$ then

$$
A u(x)=-\frac{\partial^{2} u(x)}{\partial x_{1}^{2}}+\frac{\partial^{4} u(x)}{\partial x_{2}^{4}}+u(x), \quad x=\left(x_{1}, x_{2}\right) \in \mathbb{R}^{2} .
$$

Operators of this type have been investigated by $\mathrm{H}$. Triebel in [29], by V. Shevchik in [25], and recently in [9]. Considering $\Gamma$ a regular anisotropic $d$-set with respect to the anisotropy $a=\left(\frac{4}{3}, \frac{2}{3}\right)$ we obtain:

$$
c k^{-\frac{1}{d}\left(d+\frac{2}{3}\right)} \leq \lambda_{k}\left(A^{-1} \circ t r^{\Gamma}\right) \leq C k^{-\frac{1}{d}\left(d+\frac{2}{3}\right)} \quad, \quad k \in \mathbb{N} .
$$

This is accordance with the results obtained in [9].

\section{Proofs of the results in Sect. 3}

\subsection{Proof of Theorem 3.9}

As it was already mentioned we prove here only the embedding " $\hookrightarrow$ " in (3.9).

Let $f_{\Gamma} \in L_{p}(\Gamma)$ and let $f$ be given by (3.8). We prove that $f$ is an element of $B_{p \infty^{p^{\prime}}}^{-\frac{2-d}{p^{\prime}}}\left(\mathbb{R}^{2}\right)$ and that for some $c>0$

$$
\left\|f\left|B_{p \infty}^{-\frac{2-d}{p^{\prime}}, a}\left(\mathbb{R}^{2}\right)\|\leq c\| f_{\Gamma}\right| L_{p}(\Gamma)\right\| .
$$

If $k$ is a $C^{\infty}$ function in $\mathbb{R}^{2}$ with supp $k \subset\left\{y \in \mathbb{R}^{2}:|y|_{a} \leq 1\right\}$ and $f \in S^{\prime}\left(\mathbb{R}^{2}\right)$ then we introduce the local means (cf. [30, Formula 2.4.6/1])

$$
k(t, f)(x)=\int_{\mathbb{R}^{2}} k(y) f\left(x+t^{a} y\right) d y=t^{-2} \int_{\mathbb{R}^{2}} k\left(t^{-a}(z-x)\right) f(z) d z
$$

which make sense for any $f \in S^{\prime}\left(\mathbb{R}^{2}\right)$ (appropriately interpreted).

In [10, Theorem 4.9] we obtained equivalent quasi-norms in anisotropic function spaces using local means. In case of negative smoothness we can weaken the assumptions used there; to do this we have only to repeat the 
arguments from [34, Theorem 3.1] and [33, Remark 6] and obtain for an appropriate $C^{\infty}$ function $k$ on $\mathbb{R}^{2}$ with supp $k \subset\left\{y \in \mathbb{R}^{2}:|y|_{a} \leq 1\right\}$ :

$$
\left\|f \mid B_{p \infty}^{-\frac{2-d}{p^{\prime}}, a}\left(\mathbb{R}^{2}\right)\right\| \sim \sup _{j \in \mathbb{N}_{0}}\left(2^{-j(2-d) / p^{\prime}}\left\|k\left(2^{-j}, f\right) \mid L_{p}\left(\mathbb{R}^{2}\right)\right\|\right) .
$$

Let $p<\infty$; the modification of the following estimates are obvious if $p=\infty$. Using the definition of $f$ and of $k\left(2^{-j}, f\right)$ and applying Hölder's inequality there exists a constant $c>0$ such that:

$$
\begin{aligned}
& \left|k\left(2^{-j}, f\right)(x)\right| \\
& =\left|\int_{\mathbb{R}^{2}} k(y) f\left(x+2^{-j a} y\right) d y\right| \\
& \leq 2^{2 j} \int_{\Gamma}\left|k\left(2^{j a}(\gamma-x)\right)\right|^{1 / p}\left|f_{\Gamma}(\gamma)\right| \cdot\left|k\left(2^{j a}(\gamma-x)\right)\right|^{1 / p^{\prime}} d \mu(\gamma) \\
& \leq c 2^{2 j}\left(\int_{\Gamma}\left|f_{\Gamma}(\gamma)\right|^{p}\left|k\left(2^{j a}(\gamma-x)\right)\right| d \mu(\gamma)\right)^{1 / p} \mu\left(B^{a}\left(x, 2^{-j}\right) \cap \Gamma\right)^{1 / p^{\prime}}
\end{aligned}
$$

where $B^{a}\left(x, 2^{-j}\right)=\left\{y \in \mathbb{R}^{2}:|y-x|_{a} \leq 2^{-j}\right\} \subset\left\{y \in \mathbb{R}^{2}: \mid y_{i}-\right.$ $\left.x_{i} \mid \leq c 2^{-j a_{i}}, i=1,2\right\}$. By Theorem 3.4/(i) $B^{a}\left(x, 2^{-j}\right)$ has a nonempty intersection with at most $N$ rectangles $R_{j l}\left(l=1, \ldots, N_{j}\right)$ where $N$ is independent of $j$. Using (3.5) we get $\mu\left(B^{a}\left(x, 2^{-j}\right) \cap \Gamma\right) \leq c^{\prime} 2^{-j d}$ where $c^{\prime}>0$ is independent of $j$. It follows

$$
\left|k\left(2^{-j}, f\right)(x)\right| \leq c_{1} 2^{2 j} 2^{-j d / p^{\prime}}\left(\int_{\Gamma}\left|f_{\Gamma}(\gamma)\right|^{p}\left|k\left(2^{j a}(\gamma-x)\right)\right| d \mu(\gamma)\right)^{1 / p}
$$

Using Fubini's theorem we obtain

$$
\begin{aligned}
& \left\|k\left(2^{-j}, f\right) \mid L_{p}\left(\mathbb{R}^{2}\right)\right\| \\
& \leq c_{1} 2^{2 j} 2^{-j d / p^{\prime}}\left(\int_{\Gamma}\left|f_{\Gamma}(\gamma)\right|^{p} d \mu(\gamma) \int_{\mathbb{R}^{2}}\left|k\left(2^{j a}(\gamma-x)\right)\right| d x\right)^{1 / p} \\
& =c_{2} 2^{j(2-d) / p^{\prime}}\left\|f_{\Gamma} \mid L_{p}(\Gamma)\right\|
\end{aligned}
$$

where $c_{1}$ and $c_{2}$ are independent of $j \in \mathbb{N}_{0}$. Clearly (5.3) and (5.2) lead to (5.1).

It is easy to see that we can use the above technique also for $p=1$ and obtain $L_{1}(\Gamma) \hookrightarrow B_{1 \infty}^{0, a}\left(\mathbb{R}^{2}\right)$ but this case is not especially interesting since the dimension $d$ of $\Gamma$ completely disappears in the smoothness parameter of the $B$ - space. 


\subsection{Proof of Theorem 3.10}

As it was already mentioned we prove here only the embedding $\operatorname{tr}_{\Gamma} B_{p q}^{\frac{2-d}{p}, a}\left(\mathbb{R}^{2}\right) \hookrightarrow L_{p}(\Gamma)$.

A preparation: atomic decomposition in anisotropic function spaces. Let $a=\left(a_{1}, a_{2}\right)$ be a given two dimensional anisotropy and let $\mathbb{Z}^{2}$ be the lattice of all points in $\mathbb{R}^{2}$ with integer-valued components. If $\nu \in \mathbb{N}_{0}$ and $m=$ $\left(m_{1}, m_{2}\right) \in \mathbb{Z}^{2}$ we denote $Q_{\nu m}^{a}$ the rectangle in $\mathbb{R}^{2}$ centred at $2^{-\nu a} m=$ $\left(2^{-\nu a_{1}} m_{1}, 2^{-\nu a_{2}} m_{2}\right)$ which has sides parallel to the axes and side lengths respectively $2^{-\nu a_{1}}, 2^{-\nu a_{2}}$. Remark that $Q_{0 m}^{a}$ is a square with side length 1 . If $Q_{\nu m}^{a}$ is such a rectangle in $\mathbb{R}^{2}$ and $c>0$ then $c Q_{\nu m}^{a}$ is the rectangle in $\mathbb{R}^{2}$ concentric with $Q_{\nu m}^{a}$ and with side lengths respectively $c 2^{-\nu a_{1}}, c 2^{-\nu a_{2}}$.

If $\beta=\left(\beta_{1}, \beta_{2}\right) \in \mathbb{N}_{0}^{2}$ the derivatives $D^{\beta}$ have the usual meaning and if $x=\left(x_{1}, x_{2}\right) \in \mathbb{R}^{2}$ then $x^{\beta}=x_{1}^{\beta_{1}} x_{2}^{\beta_{2}}$. The scalar product between the anisotropy $a=\left(a_{1}, a_{2}\right)$ and $\beta$ is $a \beta=a_{1} \beta_{1}+a_{2} \beta_{2}$.

Definition 5.1 Let $s \in \mathbb{R}, 0<p \leq \infty, K, L \in \mathbb{R}, c>1$.

A function $\rho: \mathbb{R}^{2} \rightarrow \mathbb{C}$ for which there exist all derivatives $D^{\beta} \rho$ if $a \beta \leq K$ (continuous if $K \leq 0$ ) is called an anisotropic $(s, p)_{K, L}$-atom, if:

$$
\begin{gathered}
\text { supp } \rho \subset c Q_{\nu m}^{a} \text { for some } \nu \in \mathbb{N} \text { and some } m \in \mathbb{Z}^{2}, \\
\left|D^{\beta} \rho(x)\right| \leq 2^{-\nu\left(s-\frac{2}{p}\right)} 2^{\nu a \beta} \quad \text { if } a \beta \leq K, \\
\int_{\mathbb{R}^{2}} x^{\beta} \rho(x) d x=0 \quad \text { if } \quad a \beta \leq L .
\end{gathered}
$$

If conditions (5.4) and (5.5) are satisfied for $\nu=0$ then $\rho: \mathbb{R}^{2} \rightarrow \mathbb{C}$ is called an anisotropic $1_{K}$-atom.

If the atom $\rho$ is located at $Q_{\nu m}^{a}$ (that means $\operatorname{supp} \rho_{\nu m}^{a} \subset c Q_{\nu m}^{a}$ with $\nu \in \mathbb{N}_{0}, m \in \mathbb{Z}^{2}, c>1$ ) then we will write it $\rho_{\nu m}^{a}$.

We give some technical explanations. The value of the number $c>1$ in (5.4) is unimportant. It simply makes clear that at the level $\nu$ some controlled overlapping of the supports of $\rho_{\nu m}^{a}$ must be allowed. If $K \leq 0$ then (5.5) is $|\rho(x)| \leq 2^{-\nu\left(s-\frac{2}{p}\right)}$.

The moment conditions (5.6) can be reformulated as $D^{\beta} \widehat{\rho}(0)=0$ if $\quad a \beta$ $\leq L$, which shows that a sufficiently strong decay of $\widehat{\rho}$ at the origin is required. If $L<0$ then (5.6) simply means that there are no moment conditions.

The reason for the normalising factor in (5.5) is that there exists a constant $c>0$ such that for all these atoms we have $\left\|\rho \mid B_{p q}^{s, a}\left(\mathbb{R}^{2}\right)\right\| \leq c$. Hence, as 
in the isotropic case, atoms are normalised building blocks satisfying some moment conditions.

This construction generalises isotropic atoms as they are in the works of M. Frazier and B. Jawerth, see [12] and [13].

If $0<p \leq \infty$ and $0<q \leq \infty$ then $b_{p q}$ is the collection of all sequences $\lambda=\left\{\lambda_{\nu m} \in \mathbb{C}: \nu \in \mathbb{N}_{0}, m \in \mathbb{Z}^{2}\right\}$ such that

$$
\left\|\lambda \mid b_{p q}\right\|=\left(\sum_{\nu=0}^{\infty}\left(\sum_{m \in \mathbb{Z}^{2}}\left|\lambda_{\nu m}\right|^{p}\right)^{q / p}\right)^{1 / q}
$$

(with the usual modification if $p=\infty$ and/or $q=\infty$ ) is finite. Clearly $b_{p q}$ is a quasi-Banach space.

Theorem 5.2 Let $a=\left(a_{1}, a_{2}\right)$ a given anisotropy with $a_{2} \leq a_{1}$.

Let $0<p \leq \infty, 0<q \leq \infty, s \in \mathbb{R}$ and let $K, L \in \mathbb{R}$ such that $K \geq a_{1}+s$ if $s \geq 0$ and $L \geq 2\left(\frac{1}{p}-1\right)_{+}-s$.

Then $g \in S^{\prime}\left(\mathbb{R}^{2}\right)$ belongs to $B_{p q}^{s, a}\left(\mathbb{R}^{2}\right)$ if, and only if, it can be represented as

$$
g=\sum_{\nu=0}^{\infty} \sum_{m \in \mathbb{Z}^{2}} \lambda_{\nu m} \rho_{\nu m}^{a} \text {, convergence being in } S^{\prime}\left(\mathbb{R}^{2}\right),
$$

where $\rho_{\nu m}^{a}$ are anisotropic $1_{K}$-atoms $(\nu=0)$ or anisotropic $(s, p)_{K, L}$-atoms $(\nu \in \mathbb{N})$ and $\lambda \in b_{p q}$ where $\lambda=\left\{\lambda_{\nu m}: \nu \in \mathbb{N}_{0}, m \in \mathbb{Z}^{2}\right\}$.

Furthermore, inf $\left\|\lambda \mid b_{p q}\right\|$ where the infimum is taken over all admissible representations (5.7), is an equivalent quasi-norm in $B_{p q}^{s, a}\left(\mathbb{R}^{2}\right)$.

A proof is given in [10, Theorem 3.3]. We will refer to the above theorem as to the atomic decomposition theorem in anisotropic function spaces.

We return now to the proof of Theorem 3.10.

Let $a=\left(a_{1}, a_{2}\right)$ be a given anisotropy with $a_{2} \leq a_{1}$. Let $K, L$ given numbers such that $K \geq a_{1}+\frac{2-d}{p}$ and $L<0$. Let $\varphi \in S\left(\mathbb{R}^{2}\right)$.

We represent $\varphi$ using the atomic decomposition theorem in $B_{p q}^{\frac{2-d}{p}, a}\left(\mathbb{R}^{2}\right)$ :

$$
\varphi=\sum_{\nu=0}^{\infty} \sum_{m \in \mathbb{Z}^{2}} \lambda_{\nu m} \rho_{\nu m}^{a} \quad \text { convergence in } S^{\prime}\left(\mathbb{R}^{2}\right),
$$

where $\rho_{\nu m}^{a}$ is an anisotropic $1_{K^{-}}$atom $(\nu=0)$ or an anisotropic $\left(\frac{2-d}{p}, p\right)_{K, L^{-}}$ atom $(\nu \in \mathbb{N})$ and

$$
\left\|\lambda\left|b_{p q}\|\leq c\| \varphi\right| B_{p q}^{\frac{2-d}{p}, a}\left(\mathbb{R}^{2}\right)\right\|
$$


In particular (5.5) with $s=\frac{2-d}{p}$ leads to $\left|\rho_{\nu m}^{a}(x)\right| \leq 2^{\nu d / p}$.

For every $\nu \in \mathbb{N}_{0}$ we denote $\varphi_{\nu}=\sum_{m \in \mathbb{Z}^{2}} \lambda_{\nu m} \rho_{\nu m}^{a}$. Using the controlled overlapping of the supports at the level $\nu$ we have:

$$
\begin{aligned}
& \left\|\varphi_{\nu}|\Gamma| L_{p}(\Gamma)\right\|^{p}=\int_{\Gamma}\left|\sum_{m \in \mathbb{Z}^{2}} \lambda_{\nu m} \rho_{\nu m}^{a}(\gamma)\right|^{p} d \mu(\gamma) \\
\leq & c \int_{\Gamma} \sum_{m \in \mathbb{Z}^{2}}\left|\lambda_{\nu m}\right|^{p} \cdot\left|2^{\nu d / p} \tilde{\chi}_{\nu m}(\gamma)\right|^{p} d \mu(\gamma) \leq c^{\prime} \sum_{m \in \mathbb{Z}^{2}}\left|\lambda_{\nu m}\right|^{p}(5.9)
\end{aligned}
$$

where in the calculation above we denoted $\widetilde{\chi}_{\nu m}$ the characteristic function of the rectangle $c Q_{\nu m}^{a}$ where $\rho_{\nu m}^{a}$ is supported.

Applying now the triangle inequality for the $L_{p^{-}}$norm if $p \geq 1$ or the $p$ - triangle inequality if $0<p<1$ and using the restriction on $q$ we obtain from (5.8) and (5.9):

$$
\left\|\varphi|\Gamma| L_{p}(\Gamma)\right\| \leq c\left\|\lambda\left|b_{p q}\left\|\leq c^{\prime}\right\| \varphi\right| B_{p q}^{\frac{2-d}{p}, a}\left(\mathbb{R}^{2}\right)\right\|
$$

and this shows, after a standard completion argument, that $\operatorname{tr}_{\Gamma}$ is a well defined linear and bounded operator from $B_{p q}^{\frac{2-d}{p}, a}\left(\mathbb{R}^{2}\right)$ into $L_{p}(\Gamma)$.

It turns out that the embedding $\operatorname{tr}_{\Gamma} B_{p q}^{\frac{2-d}{p}, a}\left(\mathbb{R}^{2}\right) \hookrightarrow L_{p}(\Gamma)$ holds for all $0<p<\infty$ and all $0<q \leq \min (1, p)$.

\section{Proof of Theorem 4.1}

We start with a subsection which has a preparatory character.

\subsection{Entropy numbers for traces on regular anisotropic d-sets}

Let $B_{1}$ and $B_{2}$ two quasi-Banach spaces. The family of all linear bounded operators $U: B_{1} \rightarrow B_{2}$ is denoted by $L\left(B_{1}, B_{2}\right)$ or $L\left(B_{1}\right)$ if $B_{1}=B_{2}$.

We will assume that the reader is familiar with the definition of the entropy numbers $e_{k}(U), k \in \mathbb{N}$, of a compact map $U \in L\left(B_{1}, B_{2}\right)$. This definition is given, for example, in [4, Definition 1.3.1] where one can find also comments and historical references.

In this subsection we will obtain estimates for entropy numbers of traces on regular anisotropic $d$-sets. We think they are of independent interest since they generalise to the anisotropic case the results from [32, Theorems 20.6,22.2]. 
Theorem 6.1 Let $0<d<2$ and let $\Gamma$ be a regular anisotropic $d$-set in $\mathbb{R}^{2}$ with respect to the anisotropy $a=\left(a_{1}, a_{2}\right)$. Let $0<p_{1} \leq \infty, 0<p_{2} \leq \infty$, $0<q \leq \infty$ and $s \in \mathbb{R}$ such that

$$
\delta_{+}=s-d\left(\frac{1}{p_{1}}-\frac{1}{p_{2}}\right)_{+}>0 .
$$

Then the trace operator

$$
\operatorname{tr}_{\Gamma}: B_{p_{1} q}^{s+\frac{2-d}{p_{1}}, a}\left(\mathbb{R}^{2}\right) \rightarrow L_{p_{2}}(\Gamma)
$$

is compact and there exists a constant $C>0$ such that for all $k \in \mathbb{N}$,

$$
e_{k}\left(\operatorname{tr}_{\Gamma}: B_{p_{1} q}^{s+\frac{2-d}{p_{1}}, a}\left(\mathbb{R}^{2}\right) \rightarrow L_{p_{2}}(\Gamma)\right) \leq C k^{-\frac{s}{d}} .
$$

Comments. We want to remark that if, in addition, $\Gamma$ is proper according to Definition 3.7 then there exists a constant $c>0$ such that for all $k \in \mathbb{N}$,

$$
c k^{-\frac{s}{d}} \leq e_{k}\left(\operatorname{tr}_{\Gamma}: B_{p_{1} q}^{s+\frac{2-d}{p_{1}}, a}\left(\mathbb{R}^{2}\right) \rightarrow L_{p_{2}}(\Gamma)\right) .
$$

To prove (6.4) one has to use the technique from [32, Theorem 20.6] adapted to our anisotropic situation. But we do not go into further details here. The interested reader can find a proof of (6.4) in [8, Theorem 3.13].

We want to remark also that the assumption (6.1) is crucial for the compactness of the operator in (6.2) and that this assumption cannot be weakened by $\delta_{+}=0$, see [32, Sect. 20.7].

Proof. (Theorem 6.1). We need some prerequisites. If $M \in \mathbb{N}$ and $0<$ $p \leq \infty$ then $l_{p}^{M}$ has the usual meaning, see for example [4, Sect. 3.2.1].

Let $d>0, \delta \geq 0$ and let $\left(M_{\nu}\right)_{\nu \in \mathbb{N}_{0}}$ be a sequence of natural numbers. We will assume that there exist two positive numbers $c_{1}$ and $c_{2}$ with

$$
c_{1} 2^{\nu d} \leq M_{\nu} \leq c_{2} 2^{\nu d} \text { for every } \quad \nu \in \mathbb{N}_{0} .
$$

Let $0<p \leq \infty$ and $0<q \leq \infty$. Then $l_{q}\left(2^{\nu \delta} l_{p}^{M_{\nu}}\right)$ denotes the linear space of all complex sequences $b=\left\{b_{\nu i}: \nu \in \mathbb{N}_{0}, i=1, \ldots, N_{\nu}\right\}$ endowed with the quasi-norm

$$
\left\|b \mid l_{q}\left(2^{\nu \delta} l_{p}^{M_{\nu}}\right)\right\|=\left(\sum_{\nu=0}^{\infty}\left(\sum_{i=1}^{M_{\nu}} 2^{\nu \delta p}\left|b_{\nu i}\right|^{p}\right)^{q / p}\right)^{1 / q}
$$

(obvious modification if $p=\infty$ and/or $q=\infty$ ).

In case of $\delta=0$ we write $l_{q}\left(l_{p}^{M_{\nu}}\right)$ and if, in addition, $p=q$ then we have the $l_{p}$ spaces with the components ordered in the given way. 
Plainly, $l_{q}\left(2^{\nu \delta} l_{p}^{M_{\nu}}\right)$ consists of dyadic blocks of spaces $l_{p}^{M_{\nu}}$ clipped together with the weights $2^{\nu \delta}$.

Let, in addition, $r \geq 0$; then by $l_{\infty}\left[2^{j r} l_{q}\left(2^{\nu \delta} l_{p}^{M_{\nu}}\right)\right]$ we shall mean the linear space of all $l_{q}\left(2^{\nu \delta} l_{p}^{M_{\nu}}\right)$ valued sequences $b=\left\{b^{j}: j \in \mathbb{N}_{0}\right\}$ endowed with the quasi-norm

$$
\left\|b\left|l_{\infty}\left[2^{j r} l_{q}\left(2^{\nu \delta} l_{p}^{M_{\nu}}\right)\right]\left\|=\sup _{j \in \mathbb{N}_{0}} 2^{j r}\right\| b^{j}\right| l_{q}\left(2^{\nu \delta} l_{p}^{M_{\nu}}\right)\right\| .
$$

Theorem 6.2 ([32, Theorem 9.2]) Let $d>0, \delta>0, r_{1}, r_{2}>0$ and $M_{\nu}$ with (6.5).

Let $0<p_{1} \leq p_{2} \leq \infty, 0<q_{1}, q_{2} \leq \infty$ and let $e_{k}$ be the entropy numbers of the identity map

$$
i d: l_{\infty}\left[2^{j r_{1}} l_{q_{1}}\left(2^{\nu \delta} l_{p_{1}}^{M_{\nu}}\right)\right] \rightarrow l_{\infty}\left[2^{j r_{2}} l_{q_{2}}\left(2^{\nu \delta} l_{p_{2}}^{M_{\nu}}\right)\right] .
$$

Then there exist two constants $c, C>0$ such that for all $k \in \mathbb{N}$,

$$
c k^{-\frac{\delta}{d}+\frac{1}{p_{2}}-\frac{1}{p_{1}}} \leq e_{k} \leq C k^{-\frac{\delta}{d}+\frac{1}{p_{2}}-\frac{1}{p_{1}}} .
$$

We return now to the proof of the estimate (6.3). Let $s_{1}=s+\frac{2-d}{p_{1}}$.

We first assume $0<p_{1} \leq p_{2} \leq \infty$. Replacing (if necessary) $|\cdot|_{a}$ by an equivalent anisotropic distance function we may assume that $\left\{x \in \mathbb{R}^{2}\right.$ : $\left.|x|_{a} \leq 2\right\} \subset[-\pi, \pi]^{2}$. So we can use the subatomic decomposition theorem in anisotropic function spaces, see [10, Theorem 3.7], and decompose any $f \in B_{p_{1} q}^{s_{1}, a}\left(\mathbb{R}^{2}\right)$ as

$$
f=\sum_{\beta \in \mathbb{N}_{0}^{2}} \sum_{\nu=0}^{\infty} \sum_{m \in \mathbb{Z}^{2}} \lambda_{\nu m}^{\beta} 2^{-\nu\left(s_{1}-\frac{2}{p}\right)} \psi^{\beta}\left(2^{\nu a} x-m\right)
$$

where $\psi$ is a $C^{\infty}$ function on $\mathbb{R}^{2}$ with supp $\psi \subset 2^{a} Q_{00}^{a}$ and $\sum_{m \in \mathbb{Z}^{2}} \psi(x-$ $m)=1$ if $x \in \mathbb{R}^{2}, \psi^{\beta}(x)=x^{\beta} \psi(x)$ and

$$
\sup _{\beta \in \mathbb{N}_{0}^{2}} 2^{r_{1} a \beta}\left\|\lambda^{\beta}\left|b_{p_{1} q}\|\leq c\| f\right| B_{p_{1} q}^{s_{1}, a}\left(\mathbb{R}^{2}\right)\right\|
$$

with $r_{1}>0$ large and $\lambda^{\beta}=\left\{\lambda_{\nu m}^{\beta}: \nu \in \mathbb{N}_{0}, m \in \mathbb{Z}^{2}\right\}$.

We denoted $Q_{00}^{a}$ the square of side length 1 having sides parallel to the axes which is centred at the origin and $2^{a} Q_{00}^{a}$ the rectangle concentric with $Q_{00}^{a}$ having side lengths $2^{a_{1}}$ and $2^{a_{2}}$.

For $\nu \in \mathbb{N}_{0}$ and $m \in \mathbb{Z}^{2}$ let $Q_{\nu m}^{a}$ be the rectangles from Definition 5.1. Let

$$
\lambda^{\beta, \Gamma}=\left\{\lambda_{\nu m}^{\beta}: \nu \in \mathbb{N}_{0}, m \in \mathbb{Z}^{2}, C Q_{\nu m}^{a} \cap \Gamma \neq \emptyset\right\}
$$


where we may assume that $C>1$ is fixed and sufficiently large such that all what follows is justified.

For a fixed $\nu \in \mathbb{N}_{0}$ let $M_{\nu}$ be the number of the rectangles $Q_{\nu m}^{a}$ such that $C Q_{\nu m}^{a} \cap \Gamma \neq \emptyset$. By Theorem 3.4/(i) it follows that there exist two constants $c_{1}, c_{2}>0$, independent of $\nu$, with

$$
c_{1} 2^{\nu d} \leq M_{\nu} \leq c_{2} 2^{\nu d}
$$

and this coincides with (6.5).

Let $l_{q}\left(2^{\nu \delta} l_{p}^{M_{\nu}}\right)$ and $l_{\infty}\left[2^{r_{1} a \beta} l_{q}\left(2^{\nu \delta} l_{p}^{M_{\nu}}\right)\right]$ be the sequence spaces introduced in (6.6) and (6.7) adapted to our present situation where $\delta=$ $s-d\left(\frac{1}{p_{1}}-\frac{1}{p_{2}}\right)$ and $r_{1}$ and $M_{\nu}$ have the same meaning as in (6.9) and (6.11) and the $l_{\infty}$ norm is now modified by sup .

Let us define the (non-linear) operator

$$
\beta \in \mathbb{N}_{0}{ }^{2}
$$

$$
U: B_{p_{1} q}^{s_{1}, a}\left(\mathbb{R}^{2}\right) \rightarrow l_{\infty}\left[2^{r_{1} a \beta} l_{q}\left(2^{\nu \delta} l_{p_{1}}^{M_{\nu}}\right)\right] \quad \text { by } \quad U f=\left\{\theta^{\beta, \Gamma}: \beta \in \mathbb{N}_{0}{ }^{2}\right\}
$$

with

$$
\theta^{\beta, \Gamma}=\left\{2^{-\nu \delta} \lambda_{\nu m}^{\beta}: \nu \in \mathbb{N}_{0}, m \in \mathbb{Z}^{2}, C Q_{\nu m}^{a} \cap \Gamma \neq \emptyset\right\}
$$

where $f$ is given by (6.8) with (6.9). By (6.9) it follows that $U$ is a bounded map.

Let $r_{2}>0$ at our disposal. We define $V: l_{\infty}\left[2^{r_{2} a \beta} l_{q}\left(2^{\nu \delta} l_{p_{1}}^{M_{\nu}}\right)\right] \rightarrow$ $L_{p_{2}}(\Gamma)$ by

$$
V(\eta)(\gamma)=\sum_{\beta \in \mathbb{N}_{0}^{2}} \sum_{\nu=0}^{\infty} \sum_{m} \eta_{\nu m}^{\beta} 2^{\nu d / p_{2}} \psi^{\beta}\left(2^{\nu a} \gamma-m\right) \quad \text { where } \quad \gamma \in \Gamma,
$$

the sum over $m$ in (6.12) being taken according to (6.10) now with $\eta_{\nu m}^{\beta}$ in place of $\lambda_{\nu m}^{\beta}$.

By Theorem 3.4/(i) and (3.5) there exists a constant $c>0$ independent of $\nu \in \mathbb{N}_{0}$ with $\mu\left(C Q_{\nu m}^{a} \cap \Gamma\right) \leq c 2^{-\nu d}$. Hence we have for fixed $\beta \in \mathbb{N}_{0}^{2}$ and $\nu \in \mathbb{N}_{0}$ :

$$
\left\|\sum_{m} \eta_{\nu m}^{\beta} 2^{\nu d / p_{2}} \psi^{\beta}\left(2^{\nu a} \cdot-m\right) \mid L_{p_{2}}(\Gamma)\right\| \leq c^{a \beta}\left(\sum_{m}\left|\eta_{\nu m}^{\beta}\right|^{p_{2}}\right)^{1 / p_{2}}
$$

where $c>1$ is independent of $\beta$. Let $\overline{p_{2}}=\min \left(1, p_{2}\right)$. Then

$$
\begin{aligned}
& \left\|\sum_{\nu=0}^{\infty} \sum_{m} \eta_{\nu m}^{\beta} 2^{\nu d / p_{2}} \psi^{\beta}\left(2^{\nu a} \cdot-m\right) \mid L_{p_{2}}(\Gamma)\right\| \|^{\overline{p_{2}}} \\
\leq & \sum_{\nu=0}^{\infty}\left(c^{a \beta}\left(\sum_{m}\left|\eta_{\nu m}^{\beta}\right|^{p_{2}}\right)^{1 / p_{2}}\right)^{\overline{p_{2}}}=c^{a \beta \overline{p_{2}}}\left\|\eta^{\beta} \mid l_{\overline{p_{2}}}\left(l_{p_{2}}^{M_{\nu}}\right)\right\|^{\overline{p_{2}}}
\end{aligned}
$$


where $\eta^{\beta}=\left\{\eta_{\nu m}^{\beta}: \nu \in \mathbb{N}_{0}, m \in \mathbb{Z}^{2}\right\}$ and $c>1$ is independent of $\beta$. Since $r_{2}>0$ is at our disposal it follows from

$$
\begin{aligned}
& \left\|V(\eta) \mid L_{p_{2}}(\Gamma)\right\|^{\overline{p_{2}}} \\
& \leq \sum_{\beta \in \mathbb{N}_{0}^{2}} c^{a \beta \overline{p_{2}}} 2^{-r_{2} a \beta \overline{p_{2}}} \sup _{\beta \in \mathbb{N}_{0}^{2}}\left(2^{r_{2} a \beta}\left\|\eta^{\beta} \mid l_{\overline{p_{2}}}\left(l_{p_{2}}^{M_{\nu}}\right)\right\|\right)^{\overline{p_{2}}} \\
& \leq c^{\prime}\left\|\eta \mid l_{\infty}\left[2^{r_{2} a \beta} l_{\overline{p_{2}}}\left(l_{p_{2}}^{M_{\nu}}\right)\right]\right\|^{\overline{p_{2}}}
\end{aligned}
$$

that $V$ is linear and bounded. With $\delta=s-d\left(\frac{1}{p_{1}}-\frac{1}{p_{2}}\right)$ we have the decomposition

$$
\operatorname{tr}_{\Gamma}=V \circ i d \circ U: B_{p_{1} q}^{s_{1}, a}\left(\mathbb{R}^{2}\right) \rightarrow L_{p_{2}}(\Gamma)
$$

where

$$
i d: l_{\infty}\left[2^{r_{1} a \beta} l_{q}\left(2^{\nu \delta} l_{p_{1}}^{M_{\nu}}\right)\right] \rightarrow l_{\infty}\left[2^{r_{2} a \beta} l_{\overline{p_{2}}}\left(l_{p_{2}}^{M_{\nu}}\right)\right]
$$

is the identity operator and the final outcome is independent of ambiguities in the non-linear construction of $U$.

Recall that if $B_{1}, B_{2}, B_{3}$ are quasi-Banach spaces, if $X \in L\left(B_{1}, B_{2}\right)$ and $R \in L\left(B_{2}, B_{3}\right)$ then for all $k, l \in \mathbb{N}$,

$$
e_{k+l-1}(R \circ X) \leq e_{k}(R) e_{l}(X),
$$

see [4, Lemma 1.3.1/1]. This is the so-called multiplication property for entropy numbers.

Using (6.14) and Theorem 6.2 it follows from (6.13) that there exists a constant $c>0$ such that for all $k \in \mathbb{N}$

$$
e_{k}\left(\operatorname{tr}_{\Gamma}: B_{p_{1} q}^{s_{1}, a}\left(\mathbb{R}^{2}\right) \rightarrow L_{p_{2}}(\Gamma)\right) \leq c k^{-\frac{\delta}{d}+\frac{1}{p_{2}}-\frac{1}{p_{1}}}
$$

and this completes the proof if $0<p_{1} \leq p_{2} \leq \infty$ since we have only to insert (6.1) in (6.15).

Let now $0<p_{2}<p_{1} \leq \infty$. The desired estimate follows now from the above considerations with $p_{1}=p_{2}$ and the embedding $L_{p_{1}}(\Gamma) \hookrightarrow L_{p_{2}}(\Gamma)$.

\subsection{Proof of Theorem 4.1/(i)}

Step 1. Equivalent norms in $W_{2}^{\left(t_{1}, t_{2}\right)}\left(\mathbb{R}^{2}\right)=W_{2}^{t, a}\left(\mathbb{R}^{2}\right)$

Lemma 6.3 Let $t_{1}, t_{2} \in \mathbb{N}$ and $A$ the operator from (4.3).

1. There exists a constant $c>0$ such that $(A u, u)_{L_{2}\left(\mathbb{R}^{2}\right)} \geq c\left\|u \mid L_{2}\left(\mathbb{R}^{2}\right)\right\|$ for any $u \in L_{2}\left(\mathbb{R}^{2}\right)$.

2. There exist two constants $c_{1}, c_{2}>0$ such that

$$
c_{1}\left\|u\left|W_{2}^{t, a}\left(\mathbb{R}^{2}\right)\left\|^{2} \leq(A u, u)_{L_{2}\left(\mathbb{R}^{2}\right)} \leq c_{2}\right\| u\right| W_{2}^{t, a}\left(\mathbb{R}^{2}\right)\right\|^{2} .
$$


Proof. of Lemma 6.3. Let $\varphi \in C_{0}^{\infty}\left(\mathbb{R}^{2}\right)$. After integration by parts we have

$$
(A \varphi, \varphi)_{L_{2}\left(\mathbb{R}^{2}\right)}=\int_{\mathbb{R}^{2}}\left(\left|\frac{\partial^{t_{1}} \varphi(x)}{\partial x_{1}^{t_{1}}}\right|^{2}+\left|\frac{\partial^{t_{2}} \varphi(x)}{\partial x_{2}^{t_{2}}}\right|^{2}+|\varphi(x)|^{2}\right) d x
$$

and the conclusion of the lemma follows immediately using the density of $C_{0}^{\infty}\left(\mathbb{R}^{2}\right)$ in $L_{2}\left(\mathbb{R}^{2}\right)$ and in $W_{2}^{t, a}\left(\mathbb{R}^{2}\right)$, and the definition of the space $W_{2}^{t, a}\left(\mathbb{R}^{2}\right)$, see (2.1).

Step 2. By Lemma 6.3 the operator $A$ is positive definite as an operator acting in $L_{2}\left(\mathbb{R}^{2}\right)$ and we may fix the norm in $W_{2}^{t, a}\left(\mathbb{R}^{2}\right)$ by $\left\|A^{1 / 2}(\cdot) \mid L_{2}\left(\mathbb{R}^{2}\right)\right\|$ and a corresponding scalar product.

Since $t_{1}, t_{2} \in \mathbb{N}$ it follows that the mean smoothness $t$, see (2.2), satisfies $t \geq 1>\frac{2-d}{2}$. Using the elementary embedding $W_{2}^{t, a}\left(\mathbb{R}^{2}\right) \hookrightarrow B_{21}^{\frac{2-d}{2}, a}\left(\mathbb{R}^{2}\right)$ and (3.10) there exists a constant $c>0$ such that

$$
\left\|\operatorname{tr}_{\Gamma} f\left|L_{2}(\Gamma)\|\leq c\| f\right| W_{2}^{t, a}\left(\mathbb{R}^{2}\right)\right\| \quad \text { for any } \quad f \in W_{2}^{t, a}\left(\mathbb{R}^{2}\right) .
$$

Defining

$$
q(f, g)=\int_{\Gamma} f(\gamma) \overline{g(\gamma)} d \mu(\gamma) \quad \text { for any } \quad f, g \in W_{2}^{t, a}\left(\mathbb{R}^{2}\right),
$$

it is clear that $q(\cdot, \cdot)$ is a non-negative quadratic form in $W_{2}^{t, a}\left(\mathbb{R}^{2}\right)$. Then there exists a non-negative and self adjoint operator $T$ uniquely determined such that

$$
q(f, g)=(T f, g)_{W_{2}^{t, a}\left(\mathbb{R}^{2}\right)} \quad \text { for any } \quad f, g \in W_{2}^{t, a}\left(\mathbb{R}^{2}\right),
$$

see for example [31, p. 91] . Furthermore,

$$
\left\|\operatorname{tr}_{\Gamma} f\left|L_{2}(\Gamma)\|=\| \sqrt{T} f\right| W_{2}^{t, a}\left(\mathbb{R}^{2}\right)\right\|
$$

where $\sqrt{T}=T^{1 / 2}$ and this proves (4.5).

So it remains to prove that the above operator is the same as in (4.4). Let $f \in W_{2}^{t, a}\left(\mathbb{R}^{2}\right)$ and $\varphi \in D\left(\mathbb{R}^{2}\right)$. Then

$$
\begin{aligned}
\int_{\Gamma} f(\gamma) \overline{\varphi(\gamma)} d \mu(\gamma) & =(T f, \varphi)_{W_{2}^{t, a}\left(\mathbb{R}^{2}\right)}=\left(A^{1 / 2} T f, A^{1 / 2} \varphi\right)_{L_{2}\left(\mathbb{R}^{2}\right)} \\
& =(A T f, \varphi)_{L_{2}\left(\mathbb{R}^{2}\right)}
\end{aligned}
$$

the second equality in (6.18) being justified by the fact that we fixed the scalar product in $W_{2}^{t, a}\left(\mathbb{R}^{2}\right)$ by

$$
(u, v)_{W_{2}^{t, a}\left(\mathbb{R}^{2}\right)}=\left(A^{1 / 2} u, A^{1 / 2} v\right)_{L_{2}\left(\mathbb{R}^{2}\right)} .
$$

Considered as a dual pairing in $\left(D\left(\mathbb{R}^{2}\right), D^{\prime}\left(\mathbb{R}^{2}\right)\right)$ we obtain $A T f=\operatorname{tr}^{\Gamma} f$ and (4.6) follows, see also [32, Theorem 27.15/Step 1].

This completes the first part of the proof of Theorem 4.1. 


\subsection{Proof of the estimate (4.7)}

Step 1. Entropy numbers and eigenvalues.

Let $B$ be a complex quasi-Banach space and $U \in L(B)$ a compact map.

We know from [4, Theorem 1.2] that the spectrum of $U$, apart from the point 0 , consists solely of eigenvalues of finite algebraic multiplicity: let $\left\{\lambda_{k}(U): k \in \mathbb{N}\right\}$ be the sequence of all non-zero eigenvalues of $U$, repeated according to algebraic multiplicity and ordered so that

$$
\left|\lambda_{1}(U)\right| \geq\left|\lambda_{2}(U)\right| \geq \ldots \geq 0 .
$$

If $U$ has only $m(<\infty)$ distinct eigenvalues and $M$ is the sum of their algebraic multiplicities, we put $\lambda_{k}(U)=0$ for $k>M$.

The following connection between the eigenvalues of the operator $U$ and its entropy numbers will be used:

Let $U$ and $\left\{\lambda_{k}(U): k \in \mathbb{N}\right\}$ as above. Then

$$
\left|\lambda_{k}(U)\right| \leq \sqrt{2} e_{k}(U) .
$$

This result was proved by B. Carl in [2], see also [4, Theorem 1.3.4].

Step 2. The operator $T$ acting in $W_{2}^{t, a}\left(\mathbb{R}^{2}\right)$ can be factored by $T=i d_{3} \circ A^{-1} \circ i d_{2} \circ \operatorname{tr}_{\Gamma} \circ i d_{1}$ where

$$
\begin{aligned}
& i d_{1}: W_{2}^{t, a}\left(\mathbb{R}^{2}\right) \quad \rightarrow \quad B_{21}^{\frac{2-d}{2}, a}\left(\mathbb{R}^{2}\right) \\
& \operatorname{tr}_{\Gamma}: B_{21}^{\frac{2-d}{2}, a}\left(\mathbb{R}^{2}\right) \rightarrow \quad L_{2}(\Gamma) \\
& i d_{2}: L_{2}(\Gamma) \quad \rightarrow \quad B_{2 \infty}^{-\frac{2-d}{2}, a}\left(\mathbb{R}^{2}\right) \\
& A^{-1}: B_{2 \infty}^{-\frac{2-d}{2}, a}\left(\mathbb{R}^{2}\right) \rightarrow \quad B_{2 \infty}^{2 t-\frac{2-d}{2}, a}\left(\mathbb{R}^{2}\right) \\
& i d_{3}: B_{2 \infty}^{2 t-\frac{2-d}{2}, a}\left(\mathbb{R}^{2}\right) \rightarrow \quad W_{2}^{t, a}\left(\mathbb{R}^{2}\right) .
\end{aligned}
$$

Since $t \geq 1>\frac{2-d}{2}$ the embedding $i d_{1}$ in the first line of (6.21) is the elementary embedding (2.3). Since $2 t-\frac{2-d}{2}>t$ the embedding $i d_{3}$ is also a consequence of the elementary embedding (2.3). The boundedness of $t r_{\Gamma}$ in the second line of (6.21) is justified by Theorem 3.10 and the embedding $i d_{2}$ is justified by Theorem 3.9.

The boundedness of $A^{-1}$ as indicated in the fourth line of (6.21) is a consequence of the lift property of the operator $A$, see the considerations in front of Theorem 4.1.

Step 3. Let $g \in W_{2}^{t, a}\left(\mathbb{R}^{2}\right)$ be an eigenfunction of $T$. Then it follows from (6.21) that $g$ belongs also to

$$
B_{2 \infty}^{2 t-\frac{2-d}{2}, a}\left(\mathbb{R}^{2}\right)
$$


and so it is an eigenfunction of the operator $T$ restricted to this space. Obviously the converse is also true.

Hence the root systems considered in $W_{2}^{t, a}\left(\mathbb{R}^{2}\right)$ and in $B_{2 \infty}^{2 t-\frac{2-d}{2}, a}\left(\mathbb{R}^{2}\right)$ coincide. Then the eigenvalues of $T$ considered in these spaces also coincide, inclusively their multiplicities.

Using the multiplication property for entropy numbers, see (6.14), and (6.21) there exists a constant $c>0$ such that for all $k \in \mathbb{N}$,

$$
\begin{aligned}
& e_{k}\left(T: B_{2 \infty}^{2 t-\frac{2-d}{2}, a}\left(\mathbb{R}^{2}\right) \rightarrow B_{2 \infty}^{2 t-\frac{2-d}{2}, a}\left(\mathbb{R}^{2}\right)\right) \\
& \quad \leq c e_{k}\left(t r_{\Gamma}: B_{2 \infty}^{2 t-\frac{2-d}{2}, a}\left(\mathbb{R}^{2}\right) \rightarrow L_{2}(\Gamma)\right) .
\end{aligned}
$$

Inserting in Theorem $6.1 p_{1}=p_{2}=2, q=\infty$ and $s+\frac{2-d}{2}=2 t-\frac{2-d}{2}$ we have from the last inequality

$$
e_{k}\left(T: B_{2 \infty}^{2 t-\frac{2-d}{2}, a}\left(\mathbb{R}^{2}\right) \rightarrow B_{2 \infty}^{2 t-\frac{2-d}{2}, a}\left(\mathbb{R}^{2}\right)\right) \leq c k^{-\frac{1}{d}(2 t-2+d)} .
$$

The estimate (4.7) is now a simple consequence of (6.22) using Carl's inequality (6.20).

\subsection{Proof of the estimate (4.8)}

Step 1. Approximation numbers and eigenvalues of operators acting in Hilbert spaces.

We recall the definition of the approximation numbers. Let $B_{1}, B_{2}$ be two quasi-Banach spaces and let $U \in L\left(B_{1}, B_{2}\right)$. Then given any $k \in \mathbb{N}$, the $k$ th approximation number $\alpha_{k}(U)$ of $U$ is defined by

$$
\alpha_{k}(U)=\inf \left\{\|U-L\|: L \in L\left(B_{1}, B_{2}\right), \operatorname{rank} L<k\right\}
$$

where rank $L$ is the dimension of the range of $L$. Usually the approximation numbers are denoted $a_{k}(U)$. The above notation is used only to avoid any possible confusion between these numbers and the anisotropy $a=\left(a_{1}, a_{2}\right)$.

We do not want to discuss here properties of approximation numbers, this is done in [4, Lemma 1.3.1/2], [4, Remark 1.3.1/6] and [3, Sect. II.2.3].

We only want to mention that approximation numbers have important connections with eigenvalues, the picture being clearest in a Hilbert space setting.

Theorem 6.4 Let $H$ be a Hilbert space and let $U \in L(H)$ be a compact, non-negative and self adjoint operator. Then the approximation numbers $\alpha_{k}(U)$ of $U$ coincide with its eigenvalues (ordered as in (6.19)). 
A proof can be found in [3, Sect. II.5.10], see also [4, p.21].

Step 2. If one applies Theorem 6.4 then the estimate (4.8) is covered by the next Proposition.

Proposition 6.5 Let $0<d<2$, let $\Gamma$ be a proper regular anisotropic $d$-set, and let $T$ be the compact, non-negative, self adjoint operator in $W_{2}^{t, a}\left(\mathbb{R}^{2}\right)$ defined in (4.4).

There exists a constant $c>0$ such that the approximation numbers $\alpha_{k}(\sqrt{T})$ of $\sqrt{T}=T^{1 / 2}$ can be estimated by

$$
\alpha_{k}(\sqrt{T}) \geq c k^{-\frac{1}{d}\left(\frac{d}{2}+t-1\right)} \quad, \quad k \in \mathbb{N} .
$$

Proof of Proposition 6.5. Let $x^{j, l}$ be the centre of the rectangle $R_{j l}$ of side lengths $r_{1}^{j, l}, r_{2}^{j, l}$ and let $N_{j} \sim 2^{j d}$ having the same meaning as in Theorem 3.4 .

Let $\varphi$ a non-negative $C^{\infty}$ function on $\mathbb{R}^{2}$ with support in $\left\{x \in \mathbb{R}^{2}\right.$ : $\left.\left|x_{1}\right|<1,\left|x_{2}\right|<1\right\}$.

We may assume $|\varphi(x)| \geq \delta>0$ if $\left|x_{1}\right| \leq \varkappa,\left|x_{2}\right| \leq \varkappa$ where $0<\varkappa<1$ is the number from Definition 3.7 of a proper set.

If

$$
\varphi_{j l}(x)=\varphi\left(\frac{2\left(x_{1}-x_{1}^{j, l}\right)}{r_{1}^{j, l}}, \frac{2\left(x_{2}-x_{2}^{j, l}\right)}{r_{2}^{j, l}}\right)
$$

then $\operatorname{supp} \varphi_{j l} \subset R_{j l}$. Furthermore, there exist two constants $c_{1}, c_{2}>0$ such that

$$
c_{1} 2^{-j d} \sum_{l=1}^{N_{j}}\left|c_{j l}\right|^{2} \leq\left\|\left.\sum_{l=1}^{N_{j}} c_{j l} \varphi_{j l}\left|L_{2}(\Gamma) \|^{2} \leq c_{2} 2^{-j d} \sum_{l=1}^{N_{j}}\right| c_{j l}\right|^{2}\right.
$$

for any complex numbers $c_{j l}$ and for any $j \in \mathbb{N}_{0}$ and $l=1, \ldots, N_{j}$. Indeed, using (3.7) we have

$$
\begin{aligned}
\left\|\sum_{l=1}^{N_{j}} c_{j l} \varphi_{j l} \mid L_{2}(\Gamma)\right\|^{2} & =\sum_{l=1}^{N_{j}} \int_{\Gamma \cap R_{j l}}\left|c_{j l}\right|^{2}\left|\varphi_{j l}(\gamma)\right|^{2} d \mu(\gamma) \\
& \geq \sum_{l=1}^{N_{j}}\left|c_{j l}\right|^{2} \delta^{2} \mu\left(\Gamma \cap \varkappa R_{j l}\right) \geq c 2^{-j d} \sum_{l=1}^{N_{j}}\left|c_{j l}\right|^{2}
\end{aligned}
$$

and this is the first inequality in (6.24). The second part of (6.24) is a simple consequence of (3.5). 
Remark now that if $j \in \mathbb{N}_{0}$ is fixed and $l \in\left\{1, \ldots, N_{j}\right\}$ then $2^{-j(t-1)} \varphi_{j l}$ is an anisotropic $(t, 2)$ atom in $W_{2}^{t, a}\left(\mathbb{R}^{2}\right)$. Hence, using the atomic decomposition theorem with $p=q=2$ we have

$$
\left\|g_{j} \mid W_{2}^{t, a}\left(\mathbb{R}^{2}\right)\right\| \leq c 2^{j(t-1)}\left(\sum_{l=1}^{N_{j}}\left|c_{j l}\right|^{2}\right)^{1 / 2}
$$

for any function $g_{j}$ of type

$$
g_{j}=\sum_{l=1}^{N_{j}} c_{j l} \varphi_{j l}=2^{j(t-1)} \sum_{l=1}^{N_{j}} c_{j l}\left(2^{-j(t-1)} \varphi_{j l}\right),
$$

the constant $c>0$ in (6.25) being independent of $j, l$ and of the complex numbers $c_{j l}$.

By (6.17), (6.24) and (6.25) we find a constant $c_{0}$ such that

$$
\begin{aligned}
\left\|\sqrt{T} g_{j} \mid W_{2}^{t, a}\left(\mathbb{R}^{2}\right)\right\| & \sim 2^{-j \frac{d}{2}}\left(\sum_{l=1}^{N_{j}}\left|c_{j l}\right|^{2}\right)^{1 / 2} \\
& \geq c_{0} 2^{-j\left(\frac{d}{2}+t-1\right)}\left\|g_{j} \mid W_{2}^{t, a}\left(\mathbb{R}^{2}\right)\right\| .
\end{aligned}
$$

There exists an operator $L=L\left(N_{j}\right)$ in $W_{2}^{t, a}\left(\mathbb{R}^{2}\right)$ with $\operatorname{rank} L<N_{j}$ such that

$$
\alpha_{N_{j}}(\sqrt{T}) \geq\|\sqrt{T}-L\|-\frac{1}{2} c_{0} 2^{-j\left(\frac{d}{2}+t-1\right)}
$$

where $c_{0}$ is the number from the last line in (6.27).

We may assume that the dimension of the span of the admitted functions $g_{j}$ in (6.26) is larger than $N_{j} \sim 2^{j d}$.

Then there exists a function $g_{j}$ of type (6.26) in $W_{2}^{t, a}\left(\mathbb{R}^{2}\right)$ such that $\left\|g_{j} \mid W_{2}^{t, a}\left(\mathbb{R}^{2}\right)\right\|=1$ and $L g_{j}=0$. It follows

$$
\begin{aligned}
\alpha_{N_{j}}(\sqrt{T}) & \geq\left\|\sqrt{T} g_{j}-L g_{j} \mid W_{2}^{t, a}\left(\mathbb{R}^{2}\right)\right\|-\frac{1}{2} c_{0} 2^{-j\left(\frac{d}{2}+t-1\right)} \\
& \geq c_{0} 2^{-j\left(\frac{d}{2}+t-1\right)}
\end{aligned}
$$

where $c_{0}>0$ is independent of $j$. Using elementary properties of the approximation numbers it is easy to see that (6.28) implies (6.23) and this completes the proof of the theorem.

Acknowledgements. This work was partly supported by the DFG Graduiertenkolleg "Analytical and stochastical structures and systems" at the University of Jena and partly by the DFG project Ja522/7-1. We are deeply indebted to Professor Hans Triebel for stimulating conversations. 


\section{References}

1. O. V. Besov, V. P. Il'in and S. M. Nikol'skij, Integral representations of functions and embedding theorems (in Russian), Nauka, Moscow, 1975

2. B. Carl, 'Entropy numbers, $s$ - numbers and eigenvalue problems', J. Funct. Anal. 41 (1981), 290-306

3. D. E. Edmunds and W. D. Evans, Spectral theory and differential operators, Oxford University Press, 1987

4. D. E. Edmunds and H. Triebel, Function spaces, entropy numbers, differential operators, Cambridge University Press, 1996

5. D. E. Edmunds and H. Triebel, 'Spectral theory for isotropic fractal drums', C. R. Acad. Sci. Paris 326 (1998), 1269-1274

6. K. J. Falconer, The geometry of fractal sets, Cambridge University Press, 1985

7. K. J. Falconer, Fractal geometry, Wiley, Chichester, 1990

8. W. Farkas, Anisotropic function spaces, fractals, and spectra of some elliptic and semi - elliptic differential operators, Ph.D. Thesis, University of Jena, 1998

9. W. Farkas, 'The behaviour of the eigenvalues for a class of operators related to some self - affine fractals in $\mathbb{R}^{2}$, Z. Anal. Anwendungen 18 (1999), 875-893

10. W. Farkas, 'Atomic and subatomic decompositions in anisotropic function spaces', Math. Nachr. 209 (2000), 83-113

11. W. Farkas and H. Triebel, 'The distribution of eigenfrequencies of anisotropic fractal drums', J. London Math. Soc. 60 (1999), 224-236

12. M. Frazier and B. Jawerth, 'Decomposition of Besov spaces', Indiana Univ. Math. J. 34 (1985), 777-799

13. M. Frazier and B. Jawerth, 'A discrete transform and decomposition of distribution spaces', J. Funct. Anal. 93 (1990), 34-170

14. T. Fujita, 'A fractal dimension, self similarity and generalized diffusion operators', in: Probabilistic methods in mathematical physics, Academic Press, Boston, 1987, pp. 83-90

15. A. Jonsson and H. Wallin, Function spaces on subsets of $\mathbb{R}^{n}$, Math. Reports, Vol. 2, Part 1, Harwood Acad. Publ., London, 1984

16. J. Kigami and M. L. Lapidus, 'Weyl's problem and the spectral distribution of Laplacians on p.c.f. self-similar fractals', Comm. Math. Phys. 158 (1993), 93-125

17. M. L. Lapidus, 'Vibrations of fractal drums. The Riemann hypothesis, waves in fractal media, and the Weyl-Berry conjecture', in: Ordinary and partial differential equations, Pitman Research Notes in Math., 289, London, Longman Sci. Techn. 1993, pp. 126-209

18. H.-G. Leopold, 'Boundedness of anisotropic pseudo - differential operators in function spaces of Besov - Hardy - Sobolev type', Z. Anal. Anwendungen 5 (1986), 409-417

19. C. McMullen, 'The Hausdorff dimension of general Sierpinski carpets', Nagoya Math. J. 96 (1984), 1-9

20. K. Naimark and M. Solomyak, On the eigenvalue behaviour for a class of operators related to self - similar measures on $\mathbb{R}^{d}$, C. R. Acad. Sci. Paris 319 (1994), 837-842

21. K. Naimark and M. Solomyak, The eigenvalue behaviour for the boundary value problems related to self - similar measures on $\mathbb{R}^{d}$, Math. Research Letters 2 (1995), 279-298

22. S. M. Nikol'skij, Approximation of functions of several variables and embedding theorems, Springer, Berlin, 1975

23. T. Runst and W. Sickel, Sobolev spaces of fractional order, Nemytskij operators and nonlinear partial differential equations, de Gruyter, Berlin, 1996

24. H.-J. Schmeißer and H. Triebel, Topics in Fourier analysis and function spaces, Geest \& Portig, Leipzig, 1987 
25. V. Shevchik, 'Spectral properties of some semi - elliptic operators in $L_{p^{-}}$spaces' Math. Nachr. 202 (1999), 151-162

26. M. Solomyak and E. Verbitsky, 'On a spectral problem related to self - similar measures', Bull. London Math. Soc. 27 (1995), 242-248

27. H. Triebel, Interpolation theory, function spaces, differential operators, North Holland, Amsterdam, 1978

28. H. Triebel, Theory of function spaces, Geest \& Portig, Leipzig, 1983

29. H. Triebel, 'A priori estimates and boundary value problems for semi - elliptic differential equations: a model case', Comm. in partial differential equations 8 (15) (1983), $1621-1644$

30. H. Triebel, Theory of function spaces II, Birkhäuser, Basel, 1992

31. H. Triebel, Higher analysis, Barth Verlag, Leipzig, 1992

32. H. Triebel, Fractals and spectra, Birkhäuser, Basel, 1997

33. H. Triebel and H. Winkelvoß, 'A Fourier analytical characterization of the Hausdorff dimension of a closed set and of related Lebesgue spaces', Studia Math. 121 (1996), 149-166

34. H. Winkelvoß, Function spaces related to fractals. Intrinsic atomic characterisations of function spaces on domains, Ph.D. Thesis, University of Jena, 1995

35. M. Yamazaki, 'A quasi-homogeneous version of paradifferential operators, I. Boundedness on spaces of Besov type', J. Fac. Sci. Univ. Tokyo, Sect. I. A, Math. 33 (1986), 131-174 